\title{
Optical tweezers: theory and practice
}

\author{
Giuseppe Pesce $^{1, a}$, Philip H. Jones ${ }^{2}$, Onofrio M. Maragò ${ }^{3}$, Giovanni Volpe ${ }^{4}$ \\ ${ }^{1}$ Department of Physics, Università degli di Studi di Napoli "Federico II" Complesso Universitario Monte S. \\ Angelo, Via Cintia, 80126 Naples, Italy \\ 2 Department of Physics and Astronomy, University College London, Gower Street, London WC1E 6BT, \\ UK \\ 3 CNR-IPCF, Istituto Processi Chimico-Fisici, V.le F. Stagno D'Alcontres 37, 98158 Messina, Italy \\ ${ }^{4}$ Department of Physics, University of Gothenburg, 41296 Gothenburg, Sweden
}

Received: 1 July 2020 / Accepted: 6 October 2020

(C) The Author(s) 2020

\begin{abstract}
The possibility for the manipulation of many different samples using only the light from a laser beam opened the way to a variety of experiments. The technique, known as Optical Tweezers, is nowadays employed in a multitude of applications demonstrating its relevance. Since the pioneering work of Arthur Ashkin, where he used a single strongly focused laser beam, ever more complex experimental set-ups are required in order to perform novel and challenging experiments. Here we provide a comprehensive review of the theoretical background and experimental techniques. We start by giving an overview of the theory of optical forces: first, we consider optical forces in approximated regimes when the particles are much larger (ray optics) or much smaller (dipole approximation) than the light wavelength; then, we discuss the full electromagnetic theory of optical forces with a focus on T-matrix methods. Then, we describe the important aspect of Brownian motion in optical traps and its implementation in optical tweezers simulations. Finally, we provide a general description of typical experimental setups of optical tweezers and calibration techniques with particular emphasis on holographic optical tweezers.
\end{abstract}

\section{Introduction}

The ability of light to exert a force on matter was recognised as early as 1619 by Kepler [1] who first described the deflection of comet tails by the rays of the sun. However, only the inclusion of light phenomena within Maxwell's theory of electromagnetism in the late nineteenth century led to the prediction of radiation pressure along the direction of light propagation [2]. Early experiments to detect the mechanical effects of light were performed by Nichols and Hull [3] and Lebedev [4], who succeeded in detecting the radiation pressure acting on macroscopic objects using thermal light sources (electric or arc lamps) and a torsion balance. A few decades later, Beth [5] reported the first experimental observation of the torque on a macroscopic object resulting from interaction with light: he observed the deflection of a quartz wave plate suspended from a thin quartz fiber when circularly polarised light passed

We would like to dedicate this paper to the memory of Arthur Ashkin (1922-2020).

\footnotetext{
a e-mail: giuseppe.pesce2@unina.it (corresponding author)
} 
Rayleigh regime

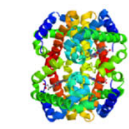

biomolecules
Intermediate regime

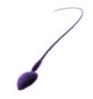

sperm cells

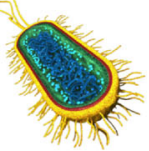

bacteria

Geometrical optics regime

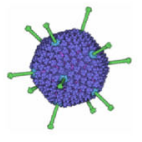

viruses

\section{(1)}
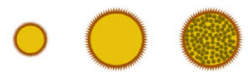

plasmonic nanoparticles

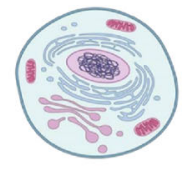

eukaryotic cells

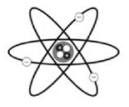

atoms

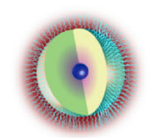

quantum dots

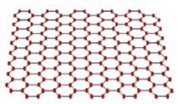

graphene

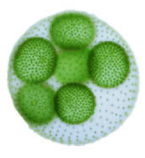

algae

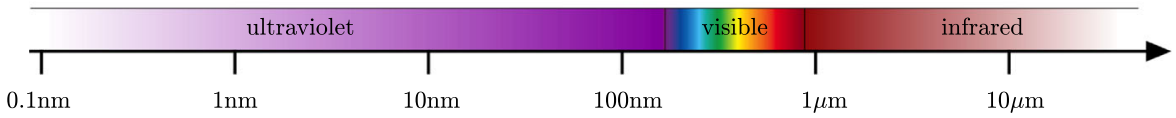

Fig. 1 Typical objects that are trapped in optical manipulation experiments. Typically, the trapping wavelength is in the visible or near-infrared spectral region

through it. The magnitude of these effects was, however, so small that they were considered insignificant for any practical use [6]. Indeed, only in 1970, and thanks to the advent of the laser [7], has it become possible to concentrate enough optical power in a small area to significantly affect the motion of microscopic particles [8] or atoms [9]. This development led to the invention of optical tweezers by Arthur Ashkin [10,11], i.e. a tightly focused laser beam that can hold and manipulate a microscopic particle in the high-intensity region that is formed at the focal spot. Optical tweezers and other optical manipulation techniques have heralded a revolution in the study of microscopic systems, spearheading new and more powerful techniques, e.g. to study biomolecules, to measure forces that act on a nanometre scale and to explore the limits of quantum mechanics. As shown in Fig. 1, the range of objects that have been optically manipulated is broad and goes from single atoms to entire mammalian cells. For the invention of optical tweezers, Arthur Ashkin was awarded the 2018 Nobel Prize in Physics (half of the prize).

From the very earliest experiments, optical trapping and optical manipulation have been applied to the biological sciences, starting by using them to trap an individual tobacco mosaic virus and Escherichia coli bacterium [12]. In the early 1990s, the use of optical force spectroscopy to characterise the mechanical properties of biomolecules and motor proteins was developed [13-15]. These biomolecular motors are ubiquitous in biology and are responsible for transport and mechanical action within the cell. Optical traps allowed biophysicists to observe the forces and dynamics of nanoscale motors at the single-molecule level, and optical force spectroscopy has led to greater understanding of the nature of these force-generating molecules $[16,17]$. Since then, optical tweezers have also proven useful in many other areas of physics [18-26], nanotechnology [27], chemistry [28], soft matter [29,30], and nanothermodynamics [31]. 
Ghislain and Webb [32] extended the capabilities of optical tweezers by devising a new kind of scanning probe microscopy using an optically trapped particle as a probe. This technique was later called photonic force microscopy [33] and provides the capability of measuring forces in the range from femtonewtons to piconewtons, a value well below that reachable with techniques that are based on microfabricated mechanical cantilevers, such as atomic force microscopy [34].

The range of optical tweezers applications has been greatly expanded by the use of advanced beam-shaping techniques, where the structure of an optical beam is altered by a diffractive optical element [35] (DOE), e.g. to produce multiple optical traps at definite positions $[23,24]$. Typically, a DOE is placed in a plane conjugate to the objective focal plane so that the complex field distribution in the trapping plane is the Fourier transform of that in the diffractive optical element plane. Often, the DOE is a computer-controlled liquid-crystal spatial light modulator [36], since this affords the potential for dynamically changing the form of the DOE, thus permitting real-time control over number, intensity and positions of the optical traps. With this technique, it is also possible to generate more complex trapping configuration, e.g. using Laguerre-Gaussian beams, which can produce torques by the transfer of orbital angular momentum [37-39].

\section{Theory of optical trapping}

The radiation force exerted by light on matter stems from the conservation of electromagnetic momentum during the scattering process. Historically, optical forces have been generally understood within strong approximations based on limiting size regimes. In fact, when the particle size is much bigger, the ray optics regime [40,41], or much smaller, the dipole approximation regime [42], than the wavelength of the light, remarkable simplifications can be made in the calculation of the force exerted. However, when the particle dimensions are comparable with the optical wavelength, the intermediate regime, a complete wave-optical modelling of the particle-light interaction is necessary for calculating the optical forces. For spherical particles, the Lorenz-Mie theory can be used to generate accurate numerical results for essentially any size and refractive index $[43,44]$. However, the situation becomes much more complex for non-spherical particles, e.g. elongated particles, optically anisotropic particles, and inhomogeneous particles [45]. Moreover, in optical trapping experiments complex objects from tens of nanometers to tens of micrometers are manipulated, and cells, biological structures, metallic, dielectric, or hybrid structures are often far from the two extreme regimes or from spherical shapes. Figure 1 provides an illustration of the range of sizes, shapes and inhomogeneous structures and compositions of objects that have been successfully manipulated in optical tweezers. Thus, in most cases full electromagnetic calculations need to be used to have a correct prediction of the optical trapping behaviour.

\subsection{Ray optics}

As is the case for many physical problems, approximate approaches are often a valuable source of physical insight that can readily and quickly give answers to complex problems. Furthermore, they have a huge pedagogical content since they are often amenable to analytical calculations. The range of validity of these approximate approaches is determined by the size parameter of the particle, defined as:

$$
\xi=2 \pi a n_{\mathrm{m}} / \lambda_{0},
$$


(a)

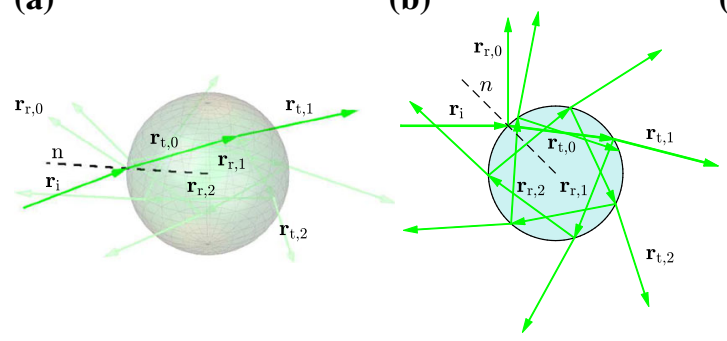

(c)

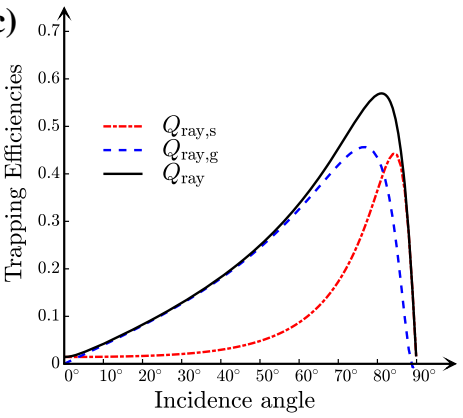

Fig. 2 Scattering and optical forces associated with a ray impinging on a sphere. a, b Multiple scattering of a light ray impinging on a sphere $\mathbf{a}$ in 3D and $\mathbf{b}$ in the incident plane. All the reflected and transmitted rays, as well as the optical force acting on the sphere lie in the plane of incidence. $\mathbf{c}$ Trapping efficiencies for a ray impinging on a glass sphere in water taking into account all scattering events

where $a$ is the characteristic size of the particle (e.g. the radius in the case of a sphere), $\lambda_{0}$ the trapping wavelength in vacuum, and $n_{\mathrm{m}}$ the refractive index of the surrounding medium (often water or air for optical manipulation experiments). Ray optics is valid when $\xi \gg 1$ and, furthermore, its accuracy increases as the size-parameter grows [40,41]. By comparison, all exact theories for non-spherical particles become unpractical when the size parameter exceeds a certain threshold. This makes ray optics an extremely useful and effective approach when dealing with large (relative to the optical wavelength) particles.

Let us consider a particle with a refractive index $n_{\mathrm{p}}$, immersed in a homogeneous, non magnetic, non-dispersive medium with refractive index $n_{\mathrm{m}}<n_{\mathrm{p}}$, and illuminated by a laser beam with vacuum wavelength $\lambda_{0}$ and hence wavenumber $k_{\mathrm{m}}=2 \pi n_{\mathrm{m}} / \lambda_{0}$ in the medium surrounding the particle. In the ray optics regime, the optical field may be described as a collection of $N$ rays, each of which is associated with a portion, $P_{\mathrm{i}}$, of the incident power, $P=\sum_{\mathrm{i}} P_{\mathrm{i}}$, and carries a linear momentum $n_{\mathrm{m}} P_{\mathrm{i}} / c$ past a fixed plane in unit time.

To understand the forces that act on a trapped microscopic particle, we start with a minimalistic model: the force due to a single ray $\mathbf{r}_{i}$ hitting a dielectric sphere at an angle of incidence $\theta_{\mathrm{i}}$ (Fig. 2a, b). When $\mathbf{r}_{\mathrm{i}}$ strikes the sphere, a small amount of power is diverted into the reflected ray $\mathbf{r}_{\mathrm{r}, 0}$, while most of the power is carried by the transmitted ray $\mathbf{r}_{\mathrm{t}, 0}$. The ray $\mathbf{r}_{\mathrm{t}, 0}$ crosses the sphere until it reaches the opposite surface, where again it is largely transmitted outside the sphere into the ray $\mathbf{r}_{\mathrm{t}, 1}$, while a further small amount is reflected inside the sphere into the ray $\mathbf{r}_{\mathrm{r}, 1}$. The ray $\mathbf{r}_{\mathrm{r}, 1}$ undergoes another scattering event as soon as it reaches the sphere boundary, and the process continues until all light has escaped from the sphere. At each scattering event, the change in momentum of the ray causes a reaction force on the center of mass of the particle. By considering these multiple reflection and refraction events, the optical force can be calculated directly as [41]:

$$
\mathbf{F}_{\text {ray }}=\frac{n_{\mathrm{m}} P_{\mathrm{i}}}{c} \hat{\mathbf{r}}_{\mathrm{i}}-\frac{n_{\mathrm{m}} P_{\mathrm{r}}}{c} \hat{\mathbf{r}}_{\mathrm{r}, 0}-\sum_{j=1}^{+\infty} \frac{n_{\mathrm{m}} P_{\mathrm{t}, j}}{c} \hat{\mathbf{r}}_{\mathrm{t}, j},
$$

where $\hat{\mathbf{r}}_{\mathrm{i}}, \hat{\mathbf{r}}_{\mathrm{r}, j}$ and $\hat{\mathbf{r}}_{\mathrm{t}, j}$ are unit vectors representing the direction of the incident ray and the $j$ th reflected and transmitted rays, respectively, calculated using Fresnel's reflection and transmission coefficients. Generally, most of the momentum transferred from the ray to the particle is due to only the first two scattering events, especially for small angle of incidence. 
The force $\mathbf{F}_{\text {ray }}$ in Eq. (2) has components only in the plane of incidence (Fig. 2b) and can be split into two perpendicular components. The component in the direction of the incoming ray $\hat{\mathbf{r}}_{\mathrm{i}}$ represents the scattering force, $\mathbf{F}_{\text {ray,s }}$, that pushes the particle in the direction of the incoming ray $\left(\hat{\mathbf{r}}_{\mathrm{i}}\right)$. The component perpendicular to the incoming ray is the gradient force, $\mathbf{F}_{\text {ray, }}$, that pulls the particle in a direction perpendicular to that of the incoming ray $\left(\hat{\mathbf{r}}_{\perp}\right)$. Dividing the force components, $F_{\text {ray,s }}$ and $F_{\text {ray,g }}$, by the rate of momentum flow in the incident ray $n_{\mathrm{i}} P_{\mathrm{i}} / c$, it is possible to define the dimensionless quantities known as the trapping efficiencies associated with the scattering and gradient forces, i.e.

$$
\begin{aligned}
Q_{\text {ray }, \mathrm{s}} & =\frac{c}{n_{\mathrm{m}} P_{\mathrm{i}}} F_{\text {ray }, \mathrm{s}}, \\
Q_{\text {ray }, \mathrm{g}} & =\frac{c}{n_{\mathrm{m}} P_{\mathrm{i}}} F_{\text {ray }, \mathrm{g}},
\end{aligned}
$$

and the total trapping efficiency as their quadrature sum

$$
Q_{\text {ray }}=\sqrt{Q_{\text {ray,s }}^{2}+Q_{\text {ray,g }}^{2}} \text {. }
$$

The trapping efficiencies permit one to quantify how effectively momentum is transferred from the light ray to the particle, the theoretical maximum value that they can reach is 2 , corresponding to complete reflection of a ray at normal incidence. Figure $2 \mathrm{c}$ shows the trapping efficiencies for a glass sphere in water; these reach up to $30 \%$ of their theoretical maximum value. $Q_{\text {ray,g }}$ grows much faster than $Q_{\text {ray,s }}$ as the angle of incidence increases and the maximum efficiencies are obtained for relatively large angles of incidence $\left(\approx 80^{\circ}\right)$. It is interesting to note also that these results are independent of the size of the sphere. The scattering and gradient efficiencies of a circularly polarised ray on a sphere were derived by Ashkin [40]:

$$
\begin{aligned}
& Q_{\text {scat }}=1+R \cos 2 \theta_{i}-T^{2} \frac{\cos \left(2 \theta_{i}-2 \theta_{r}\right)+R \cos 2 \theta_{i}}{1+R^{2}+2 R \cos 2 \theta_{r}}, \\
& Q_{\text {grad }}=R \sin 2 \theta_{i}-T^{2} \frac{\sin \left(2 \theta_{i}-2 \theta_{r}\right)+R \sin 2 \theta_{i}}{1+R^{2}+2 R \cos 2 \theta_{r}},
\end{aligned}
$$

where $R$ and $T$ are the Fresnel reflection and transmission coefficient, and $\theta_{i}$ and $\theta_{r}$ are the incidence and transmission angle relative to the scattering of the incident beam.

To model an optical trap, we must not only consider a single incident ray but all the rays constituting a highly-focused laser beam, that is a set of many rays that converge at a very large angle. This means that the total force acting on the particle is the sum of all the contributions from each ray forming the beam. For a single-beam optical trap, the focused rays will produce a restoring force proportional to the particle's displacement from an equilibrium position that lies close to the beam waist. Hence, in one direction, $x$, for very small displacements, we have a harmonic response of the type $F_{x} \approx-\kappa_{x} x$, where $\kappa_{x}$ is the spring constant or trap stiffness in the $x$ direction and the origin of the axis is taken at the trap equilibrium position. A calculation or measurement of the spring constants in the three orthogonal directions gives a calibration of the optical trap.

A geometrical optics approach can also be employed to study more complex non-spherical geometries, as long as all the characteristic dimensions of the object under study are significantly larger than the wavelength of the light used for trapping. There are, however, two major differences. First, in the case of non-spherical objects a significant torque can also appear inducing the rotation of the object. This effect is known as the windmill effect because of its analogy to the motion of a windmill, where the wind in this case is the flow of momentum 
due to the electromagnetic field [46]. The torque due to a single ray can be calculated as the difference of the angular momentum associated with the incoming ray and that associated with the outgoing rays. The total torque on the object can then be calculated as the vector sum of the torques due to each ray. Second, while for a spherical particle the radiation pressure of a plane wave, i.e. a set of parallel rays, is always directed in the propagation direction because of symmetry, for particles of anisotropic shapes the radiation pressure has a transverse component that is responsible for and optical lift effect, i.e. non-spherical particles can move transversely with respect to the incident light propagation direction $[47,48]$.

\subsection{Dipole approximation}

The dipole approximation is based on the assumption that particles can be approximated as small dipoles, and that fields are homogeneous inside the particles. This sets a range of validity that is expressed by two conditions [49]:

(i) $\xi \ll 1$,

(ii) $|m| \xi \ll 1$,

where $m=n_{\mathrm{p}} / n_{\mathrm{m}}$ is the relative refractive index between the particle, $n_{\mathrm{p}}$, and the surrounding medium. Note that the condition (ii) needs to be considered with care, especially when dealing with nanostructures that are often made of materials with high refractive index, e.g. for silicon nanoparticles $\left(n_{\mathrm{p}} \sim 3.7\right.$ at $\left.\lambda \sim 830 \mathrm{~nm}\right)$.

An incident electromagnetic field $\mathbf{E}_{\mathrm{i}}$ induces an electric dipole moment, $\mathbf{p}$, that, for sufficiently small fields, can be expressed in terms of the particle polarisability as

$$
\mathbf{p}=\alpha_{\mathrm{p}}(\omega) \mathbf{E}_{\mathrm{i}},
$$

where $\alpha_{\mathrm{p}}$ is the complex polarisability of the particle relative to the surrounding medium given by [50]

$$
\alpha_{\mathrm{p}}=\frac{\alpha_{\mathrm{CM}}}{1-i \alpha_{C M} k^{3} /\left(6 \pi \varepsilon_{0} \varepsilon_{\mathrm{m}}\right)}
$$

with $\alpha_{\mathrm{CM}}$ being the static Clausius-Mossotti polarisability, i.e.

$$
\alpha_{\mathrm{CM}}=3 V \varepsilon_{0} \varepsilon_{\mathrm{m}}\left(\frac{\varepsilon_{\mathrm{p}}-\varepsilon_{\mathrm{m}}}{\varepsilon_{\mathrm{p}}+2 \varepsilon_{\mathrm{m}}}\right),
$$

where $\varepsilon_{\mathrm{m}}$ and $\varepsilon_{\mathrm{p}}$ are the relative permittivities of the medium and particle, respectively, and $V$ is the particle volume. The complex polarisability $\alpha_{\mathrm{p}}$, which typically depends on the frequency of the electromagnetic field $\omega$, has a real part, $\alpha_{\mathrm{p}}^{\prime}$, which represents the oscillation of the dipole in phase with the field, and an imaginary part, $\alpha_{\mathrm{p}}^{\prime \prime}$ which represents its oscillation in phase quadrature.

The time-averaged optical force experienced by an electric dipole in the presence of a time-varying electric field can then be expressed in terms of cross sections and particle's polarisability [51-53]:

$$
\mathbf{F}_{\mathrm{DA}}=\frac{1}{4} \alpha_{\mathrm{p}}^{\prime} \nabla\left|E_{\mathrm{i}}\right|^{2}+\frac{n_{\mathrm{m}} \sigma_{\mathrm{ext}}}{c} \mathbf{S}_{\mathrm{i}}-\frac{1}{2} n_{\mathrm{m}} \sigma_{\mathrm{ext}} c \nabla \times \mathbf{s}_{\mathrm{d}},
$$

where $\sigma_{\text {ext }}=k \operatorname{Im}\left\{\alpha_{\mathrm{p}}\right\} / \varepsilon_{\mathrm{m}}$ is the particle extinction cross section, $\mathbf{S}_{\mathrm{i}}$ is the time-averaged Poynting vector of the incident electromagnetic field, and $\mathbf{s}_{\mathrm{d}}$ is the time-averaged spin density $[54,55]$.

The first term in Eq. (11) is the gradient force:

$$
\mathbf{F}_{\text {grad }}(\mathbf{r})=\frac{1}{4} \alpha_{\mathrm{p}}^{\prime} \nabla\left|E_{\mathrm{i}}(\mathbf{r})\right|^{2}
$$


This is the force that is responsible for confinement in optical tweezers. It arises from the potential energy of a dipole immersed in the electric field, and hence it is a conservative force. Since the intensity of the electric field is $I_{\mathrm{i}}=\frac{1}{2} c n_{\mathrm{m}}\left|E_{\mathrm{i}}\right|^{2}$, we can re-write the gradient force in terms of the gradient of intensity as

$$
\mathbf{F}_{\operatorname{grad}}(\mathbf{r})=\frac{1}{2} \frac{\alpha_{\mathrm{p}}^{\prime}}{c n_{\mathrm{m}}} \nabla I_{\mathrm{i}}(\mathbf{r}) .
$$

Therefore, particles with positive polarisability, i.e. particles whose refractive index is higher than that of the surrounding medium, are attracted towards the high-intensity region of the optical field [10], and particles with negative polarisability, i.e. particles whose refractive index is lower than that of the surrounding medium, are repelled by the high-intensity regions, such as at the focal spot of a focused beam (Fig. 3a).

Let us consider as an example an incident laser beam with a typical Gaussian intensity profile:

$$
I_{\mathrm{i}}(\rho)=I_{0} \mathrm{e}^{-\frac{2 \rho^{2}}{w_{0}^{2}}}
$$

where $\rho$ is the radial coordinate in the transverse plane, $I_{0}$ is the maximum intensity and $w_{0}$ is the laser beam waist. This intensity distribution and the corresponding optical forces on a small particle with a high relative refractive index are shown in Fig. 3b. For small displacement from the beam axis, $\rho / w_{0} \ll 1$, we can expand the profile, $I_{\mathrm{i}}(\rho) \approx I_{0}\left(1-2 \rho^{2} / w_{0}^{2}\right)$, and approximate the radial component of the optical force as an elastic force proportional and opposite to the displacement from the origin, i.e. $F_{\operatorname{grad}, \rho}(\rho)=-\kappa_{\rho} \rho$, where

$$
\kappa_{\rho}=2 \frac{\alpha_{\mathrm{p}}^{\prime}}{c n_{\mathrm{m}}} \frac{I_{0}}{w_{0}^{2}} .
$$

Equation (15) reveals that $\kappa_{\rho}$ is proportional to the electric field intensity and to the real part of the polarisability, i.e. to the particle volume for small dipolar particles. Furthermore, $\kappa_{\rho}$ is inversely proportional to the beam area, so, as may be expected, tighter focusing leads to

(a)

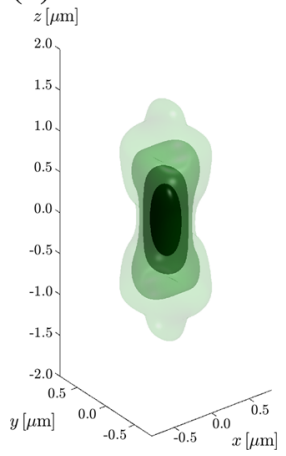

(b)

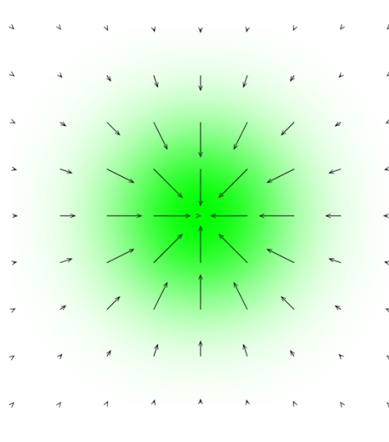

(c)

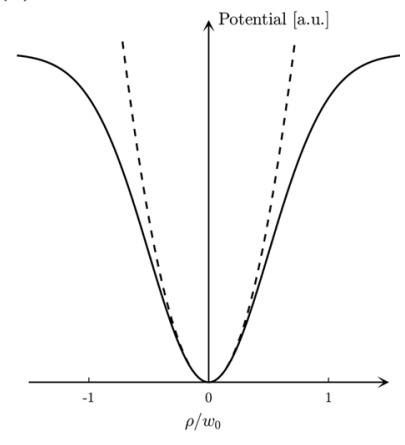

Fig. 3 Gradient forces generated by a focused Gaussian beam. a Intensity distribution at the focus of a 1.2 NA water-immersion objective for an $x$-polarised Gaussian beam with beam waist $w_{0}=4 \mathrm{~mm}$; the laser beam wavelength is $\lambda_{0}=632 \mathrm{~nm}$ and the full laser power after the aperture is $10 \mathrm{~mW}$; the iso-intensity surfaces correspond to $I(x, y, z)=50,20,10,5 \mathrm{GW} \mathrm{m}^{-2}$. b Gaussian intensity profile (background) in the transverse $x y$-plane and corresponding optical gradient forces (arrows) on a small dielectric particle whose refractive index is higher than that of the surrounding medium. c Optical potential along the radial direction (solid line) and its approximation with a harmonic potential (dashed line) 
stronger confinement. The corresponding radial potential, $U_{\mathrm{DA}}(\rho)=\frac{1}{2} \kappa_{\rho} \rho^{2}$, is plotted by the dashed line in Fig. 3c, showing that it is a good approximation to the real potential (solid line) for small particle displacements from the potential minimum. A similar analysis may be made for the axial direction, although the spring constant in this direction will be found to be weaker.

The second and third terms in Eq. (11) represent the non-conservative components of the optical force. The second term is the radiation pressure or scattering force:

$$
\mathbf{F}_{\text {scat }}(\mathbf{r})=\frac{n_{\mathrm{m}} \sigma_{\mathrm{ext}}}{c} \mathbf{S}_{\mathrm{i}}(\mathbf{r}) .
$$

It arises from the transfer of momentum from the field to the particle as a result of scattering and absorption processes, as is revealed by the fact that $\mathbf{F}_{\text {scat }}$ is proportional to the extinction cross section $\sigma_{\text {ext }}$. This force points in the direction of the Poynting vector, $\mathbf{S}_{\mathrm{i}}$ [8]. However, note that this direction not always coincides with the direction of the local field propagation $[56,57]$.

The last term in Eq. (11) is the so-called spin-curl force [53,55]:

$$
\mathbf{F}_{\text {spin }}(\mathbf{r})=-\frac{1}{2} \sigma_{\mathrm{ext}} c \nabla \times \mathbf{s}_{\mathrm{d}}(\mathbf{r})
$$

In order for this force to arise, the polarisation of the field must be non-homogeneous, which may occur, e.g. in the case of high numerical aperture focusing. This force is relatively small compared to the gradient and scattering forces and, therefore, does not usually play a major role in optical trapping experiments. However, it may yield larger effects when considering higher-order optical beams with inhomogeneous polarisation patterns such as superpositions of circularly polarised Hermite-Gaussian beams [58] or cylindrical vector beams [59-61].

Here, we represented the non-conservative components of the optical force in terms of the well-known radiation pressure plus the spin-curl force. Alternatively, the time-averaged Poynting vector can be decomposed into the sum of an orbital and spin momentum density [62] and hence the non-conservative optical forces can be related to the orbital component of the field momentum directed as the local wavevector $[55,56]$. Thus, spin-dependent optical forces occur when the Poynting vector is not directed as the local wavevector and a transverse chiral component of the force can be identified [57,63].

\subsection{Electromagnetic theory}

The intermediate regime is characterised by a size of the particle that is comparable to the optical wavelength. In the intermediate regime, the dipole and geometrical optics approximations are not strictly valid. Therefore, a full wave-optical modelling of the interaction between light and particles (i.e. light scattering) is required to calculate optical forces and torques. Starting from the laws of conservation of linear and angular momentum, it is possible to derive the resulting optical force and torque from the distribution of the scattered fields. In particular, by exploiting the conservation of linear momentum, the time-averaged optical force exerted by monochromatic light on a particle is found to be [64-67]:

$$
\mathbf{F}_{\mathrm{rad}}=\int_{S} \overline{\mathrm{T}}_{\mathrm{M}} \cdot \hat{\mathbf{n}} d S,
$$

where the integration is carried out over a surface $S$ surrounding the scattering particle. The vector $\hat{\mathbf{n}}$ is the (outwards) unit vector normal to the $S$, and $\overline{\mathrm{T}}_{\mathrm{M}}$ is the time-averaged Maxwell stress tensor which describes the mechanical interaction of an electromagnetic field with 
matter, which needs to be defined carefully [68]. For harmonic fields, this quantity is defined in terms of the total fields complex amplitudes, $\mathbf{E}_{\mathrm{t}}$ and $\mathbf{B}_{\mathrm{t}}$, as

$$
\overline{\mathrm{T}}_{\mathrm{M}}=\frac{1}{2} \varepsilon_{\mathrm{m}} \operatorname{Re}\left[\mathbf{E}_{\mathrm{t}} \otimes \mathbf{E}_{\mathrm{t}}^{*}+\frac{c^{2}}{n_{\mathrm{m}}^{2}} \mathbf{B}_{\mathrm{t}} \otimes \mathbf{B}_{\mathrm{t}}^{*}-\frac{1}{2}\left(\left|\mathbf{E}_{\mathrm{t}}\right|^{2}+\frac{c^{2}}{n_{\mathrm{m}}^{2}}\left|\mathbf{B}_{\mathrm{t}}\right|^{2}\right) \mathrm{I}\right],
$$

where $\otimes$ represents the dyadic (outer) product, I is the unit dyadic, and the fields $\mathbf{E}_{\mathrm{t}}=\mathbf{E}_{\mathrm{i}}+\mathbf{E}_{\mathrm{s}}$ and $\mathbf{B}_{\mathrm{t}}=\mathbf{B}_{\mathrm{i}}+\mathbf{B}_{\mathrm{s}}$ are the total electric and magnetic fields resulting from the superposition of the incident $\left(\mathbf{E}_{\mathrm{i}}, \mathbf{B}_{\mathrm{i}}\right)$ and scattered $\left(\mathbf{E}_{\mathrm{s}}, \mathbf{B}_{\mathrm{s}}\right)$ fields. Similarly, by exploiting the conservation of angular momentum, the time-averaged radiation torque is found to be [69]

$$
\mathbf{T}_{\mathrm{rad}}=-\int_{S}\left(\overline{\mathrm{T}}_{\mathrm{M}} \times \mathbf{r}\right) \cdot \hat{\mathbf{n}} \mathrm{d} S .
$$

In the far-field region, the expressions for the radiation force and torque can be significantly simplified. By performing the integration over a spherical surface of very large radius, $r \rightarrow$ $\infty$, fast decaying terms in the integration may be neglected and the radiation force and torque are [64-67]

$$
\begin{aligned}
\mathbf{F}_{\mathrm{rad}} & =-\frac{\varepsilon_{\mathrm{m}} r^{2}}{4} \int\left[\left|\mathbf{E}_{\mathrm{s}}\right|^{2}+\frac{c^{2}}{n_{\mathrm{m}}^{2}}\left|\mathbf{B}_{\mathrm{s}}\right|^{2}+2 \operatorname{Re}\left\{\mathbf{E}_{\mathrm{i}} \cdot \mathbf{E}_{\mathrm{s}}^{*}+\frac{c^{2}}{n_{\mathrm{m}}^{2}} \mathbf{B}_{\mathrm{i}} \cdot \mathbf{B}_{\mathrm{s}}^{*}\right\}\right] \hat{\mathbf{r}} \mathrm{d} \Omega, \\
\mathbf{T}_{\mathrm{Rad}} & =-\frac{\varepsilon_{\mathrm{m}} r^{3}}{2} \operatorname{Re}\left\{\int\left[\left(\hat{\mathbf{r}} \cdot \mathbf{E}_{\mathrm{t}}\right)\left(\mathbf{E}_{\mathrm{t}}^{*} \times \hat{\mathbf{r}}\right)+\frac{c^{2}}{n_{\mathrm{m}}^{2}}\left(\hat{\mathbf{r}} \cdot \mathbf{B}_{\mathrm{t}}\right)\left(\mathbf{B}_{\mathrm{t}}^{*} \times \hat{\mathbf{r}}\right)\right] \mathrm{d} \Omega\right\},
\end{aligned}
$$

where the integration is now carried out over the full solid angle $(\Omega=4 \pi)$. These expressions are the starting point for the electromagnetic calculations of optical forces and torques in optical trapping in the intermediate size regime as well as for inhomogeneous and nonspherical scatterers. The key point is to solve the scattering problem by calculating the scattered fields and consequently the Maxwell stress tensor, from which the optical force and torque can be found, as in Eqs. (18) and (20). However, the calculations of forces and torques in this regime can be a complicated and cumbersome procedure [45]; thus, various approaches have been developed to handle this problem [70,71]. Among the different methods, one successful approach is based on the calculation of the transition matrix (T-matrix) [45]; this is particularly useful and computationally effective because it is possible to exploit the rotation and translation properties of the T-matrix to obtain at once optical forces and torques for different positions and orientations of the trapped particles [65-67,69,72-77].

In Fig. 4, we compare the results for the transversal trapping stiffness $\kappa_{x}$ for a spherical particle held by an optical tweezers as a function of the particle radius $a$ obtained with the exact electromagnetic calculations (solid line) with those obtained with geometrical optics (dashed line) and with dipole approximation (dotted line). Interestingly, the agreement is quite good also beyond the range of particle sizes where the approximations are strictly valid.

\subsubsection{T-matrix methods}

When using T-matrix methods, the incident, scattered and internal fields are expanded in terms of vector spherical harmonics. The relation between the expansion amplitudes of the scattered and of the incident fields defines the T-matrix; thus, the scattering problem is reduced to the calculations of these coefficients through, e.g. the imposition of the boundary conditions across the surface of the particle, or by point matching numerically the fields at the surface $[11,45]$. T-matrix methods work best with objects that are highly symmetric in 


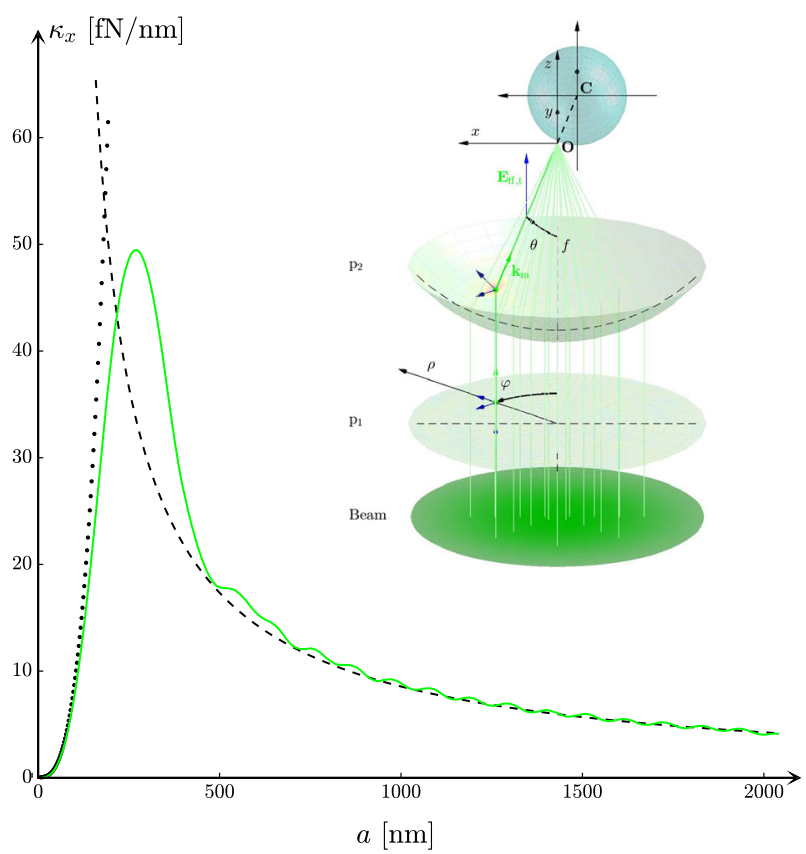

Fig. 4 Comparison between exact electromagnetic theory, geometrical optics and dipole approximation. Transverse trap stiffness produced by a $10 \mathrm{~mW}$ laser beam of wavelength $\lambda_{0}=632 \mathrm{~nm}$ focused by a $1.2 \mathrm{NA}$ objective on a dielectric sphere of radius $a\left(n_{\mathrm{p}}=1.50\right)$ in water $\left(n_{\mathrm{m}}=1.33\right)$. The solid line represents the exact electromagnetic calculation. The dipole approximation (dotted line) works for small spheres $\left(a \ll \lambda_{0}\right)$. The geometrical optics approximation (dashed line) works for large spheres $\left(a \gg \lambda_{0}\right)$. Inset: reference frames used to calculate the radiation force from a focused beam; the scattering problem is solved in the reference frame of the particle, centred at $\mathbf{C}$, while the radiation force and torque are calculated with respect to the laboratory frame centred at the laser beam focus $\mathbf{O}$

shape and composition, so one can calculate forces and torques on non-spherical objects by modelling them as clusters of small spheres $[67,75]$.

Because of the linearity of Maxwell's equations and of the boundary conditions, the scattering process can be considered in terms of a linear operator $\mathbb{T}$ so that $\mathbf{E}_{\mathrm{s}}=\mathbb{T} \mathbf{E}_{\mathrm{i}}$, where $\mathbf{E}_{\mathrm{i}}$ and $\mathbf{E}_{\mathrm{s}}$ are the incident and scattered fields, respectively. Therefore, if both $\mathbf{E}_{\mathrm{i}}$ and $\mathbf{E}_{\mathrm{s}}$ are expanded in suitable bases (not necessarily the same), it is possible to find a transition matrix $\mathbb{T}$ that relates the coefficients of such expansions, encompassing all the information about the morphology and orientation of the particle with respect to the incident field [78].

Since $\mathbf{E}_{\mathrm{i}}$ is, in general, finite at the origin, its expansion is conveniently given in terms of Bessel J-multipoles:

$$
\mathbf{E}_{\mathrm{i}}(r, \hat{\mathbf{r}})=E_{\mathrm{i}} \sum_{l=0}^{\infty} \sum_{m=-l}^{l} W_{l m}^{(1)} \mathbf{J}_{l m}^{(1)}(k r, \hat{\mathbf{r}})+W_{l m}^{(2)} \mathbf{J}_{l m}^{(2)}(k r, \hat{\mathbf{r}}),
$$

with amplitudes $W_{\mathrm{i}, l m}^{(1)}$ and $W_{\mathrm{i}, l m}^{(2)}$. The superscript " 1 " ("2") refers to multipolar components of magnetic (electric) kind, i.e. to magnetic (electric) transverse radiant modes with the components aligned along the magnetic (electric) field. Since $\mathbf{E}_{\mathrm{s}}$ must satisfy the radiation condition at infinity, it is convenient to expand it in terms of Hankel $\mathbf{H}$-multipoles: 


$$
\mathbf{E}_{\mathrm{s}}(r, \hat{\mathbf{r}})=\sum_{l=0}^{\infty} \sum_{m=-l}^{l} A_{l m}^{(1)} \mathbf{H}_{l m}^{(1)}(k r, \hat{\mathbf{r}})+A_{l m}^{(2)} \mathbf{H}_{l m}^{(2)}(k r, \hat{\mathbf{r}}),
$$

with amplitudes $A_{\mathrm{s}, l m}^{(1)}$ and $A_{\mathrm{s}, l m}^{(2)}$. These amplitudes are determined by imposing the boundary conditions across the surface of the scattering particle.

The transition matrix $\mathbb{T}=\left\{T_{l^{\prime} m^{\prime} l m}^{\left(p^{\prime} p\right)}\right\}$ of the scattering particle acts on the known multipole amplitudes of the incident field $W_{\mathrm{i}, l m}^{(p)}$, where $p=1,2$, to give the unknown amplitudes of the scattered field $A_{\mathrm{s}, l^{\prime} m^{\prime}}^{\left(p^{\prime}\right)}$, where $p^{\prime}=1,2$, i.e.

$$
A_{\mathrm{s}, l^{\prime} m^{\prime}}^{\left(p^{\prime}\right)}=\sum_{p=1,2} \sum_{l=0}^{+\infty} \sum_{m=-l}^{l} T_{l^{\prime} m^{\prime} l m}^{\left(p^{\prime} p\right)} W_{\mathrm{i}, l m}^{(p)},
$$

or, in a more compact form, $\mathbf{A}_{\mathrm{s}}=\mathbb{T} \mathbf{W}_{\mathrm{i}}$, where $\mathbf{A}_{\mathrm{s}}=\left\{A_{\mathrm{s}, l^{\prime} m^{\prime}}^{\left(p^{\prime}\right)}\right\}$ and $\mathbf{W}_{\mathrm{i}}=\left\{W_{\mathrm{i}, l m}^{(p)}\right\}$. For example, the T-matrix for a homogeneous spherical particle is diagonal, independent of $m$, and connected to the Mie coefficients $a_{l}$ and $b_{l}$, i.e. $\mathbf{A}_{\mathrm{s}}=-\mathbb{R} \mathbf{W}_{\mathrm{i}}$, where $\mathbb{R}=\left\{R_{l^{\prime} m^{\prime} l m}^{\left(p^{\prime} p\right)}\right\}$ and

$$
R_{l^{\prime} m^{\prime} l m}^{\left(p^{\prime} p\right)}= \begin{cases}b_{l} & p=p^{\prime}=1 \text { and } l=l^{\prime} \text { and } m=m^{\prime} \\ a_{l} & p=p^{\prime}=2 \text { and } l=l^{\prime} \text { and } m=m^{\prime} \\ 0 & \text { otherwise }\end{cases}
$$

\subsubsection{Optical force}

The starting point from which to calculate the optical force is Eq. (21) [67]. By substituting the expansions of the incident and scattered waves in terms of multipoles given by Eqs. (23) and (24) into Eq. (21), we obtain the expression for the radiation force along the direction of a unit vector $\hat{\mathbf{u}}$, i.e. $F_{\text {rad }}(\hat{\mathbf{u}})=\mathbf{F}_{\mathrm{rad}} \cdot \hat{\mathbf{u}}$,

$$
F_{\mathrm{rad}}(\hat{\mathbf{u}})=-\frac{\varepsilon_{\mathrm{m}} E_{\mathrm{i}}^{2}}{2 k_{\mathrm{m}}^{2}} \operatorname{Re}\left\{\sum_{p l m} \sum_{p^{\prime} l^{\prime} m^{\prime}} i^{l-l^{\prime}} I_{l m l^{\prime} m^{\prime}}^{\left(p p^{\prime}\right)}(\hat{\mathbf{u}})\left[A_{\mathrm{s}, l m}^{(p) *} A_{\mathrm{s}, l^{\prime} m^{\prime}}^{\left(p^{\prime}\right)}+W_{\mathrm{i}, l m}^{(p) *} A_{\mathrm{s}, l^{\prime} m^{\prime}}^{\left(p^{\prime}\right)}\right]\right\}
$$

where the amplitudes $A_{\mathrm{s}, l^{\prime} m^{\prime}}^{(p)}$ of the scattered field are given in terms of the elements of the T-matrix, the amplitudes $W_{\mathrm{i}, l m}^{(p) *}$ of the incident field by Eq. (25) and the integrals $I_{l m l^{\prime} m^{\prime}}^{\left(p p^{\prime}\right)}(\hat{\mathbf{u}})$ can be expressed in closed form [67] as

$$
I_{l m l^{\prime} m^{\prime}}^{\left(p p^{\prime}\right)}(\hat{\mathbf{u}})=\sqrt{\frac{4 \pi}{3}} \sum_{\mu=-1,0,1} Y_{1 \mu}^{*}(\hat{\mathbf{u}}) C_{1}\left(l^{\prime}, l ; \mu, m-\mu\right) O_{l l^{\prime}}^{\left(p p^{\prime}\right)},
$$

with

$$
O_{l l^{\prime}}^{\left(p p^{\prime}\right)}=\left\{\begin{array}{cc}
\sqrt{\frac{(l-1)(l+1)}{l(2 l+1)}} & l^{\prime}=l-1 \text { and } p=p^{\prime} \\
-\frac{1}{\sqrt{l(l+1)}} & l^{\prime}=l \text { and } p \neq p^{\prime} \\
-\sqrt{\frac{l(l+2)}{(l+1)(2 l+1)}} & l^{\prime}=l+1 \text { and } p=p^{\prime} \\
0 & \text { otherwise }
\end{array}\right.
$$


and $C_{1}\left(l^{\prime}, l ; \mu, m-\mu\right)$ are Clebsch-Gordan coefficients. The integrals $I_{l m l^{\prime} m^{\prime}}^{\left(p p^{\prime}\right)}$ obey the symmetry properties: $I_{\mu ; l m l^{\prime} m^{\prime}}^{(11)}=I_{\mu ; l m l^{\prime} m^{\prime}}^{(22)}, \quad I_{\mu ; l m l^{\prime} m^{\prime}}^{(12)}=I_{\mu ; l m l^{\prime} m^{\prime}}^{(21)}$.

It is interesting to note that the force expressed by Eq. (27) can be separated into two parts, i.e. $F_{\text {rad }}(\hat{\mathbf{u}})=-F_{\text {scat }}(\hat{\mathbf{u}})+F_{\text {ext }}(\hat{\mathbf{u}})$, where

$$
F_{\mathrm{scat}}(\hat{\mathbf{u}})=\frac{\varepsilon_{\mathrm{m}} E_{\mathrm{i}}^{2}}{2 k_{\mathrm{m}}^{2}} \operatorname{Re}\left\{\sum_{p l m} \sum_{p^{\prime} l^{\prime} m^{\prime}} A_{\mathrm{s}, l m}^{(p) *} A_{\mathrm{s}, l^{\prime} m^{\prime}}^{\left(p^{\prime}\right)} l^{l-l^{\prime}} I_{l m l^{\prime} m^{\prime}}^{\left(p p^{\prime}\right)}(\hat{\mathbf{u}})\right\}
$$

and

$$
F_{\mathrm{ext}}(\hat{\mathbf{u}})=-\frac{\varepsilon_{\mathrm{m}} E_{\mathrm{i}}^{2}}{2 k_{\mathrm{m}}^{2}} \operatorname{Re}\left\{\sum_{p l m} \sum_{p^{\prime} l^{\prime} m^{\prime}} W_{\mathrm{i}, l m}^{(p) *} A_{\mathrm{s}, l^{\prime} m^{\prime}}^{\left(p^{\prime}\right)} i^{l-l^{\prime}} I_{l m l^{\prime} m^{\prime}}^{\left(p p^{\prime}\right)}(\hat{\mathbf{u}})\right\} .
$$

$F_{\text {scat }}(\hat{\mathbf{u}})$ depends on the amplitudes $A_{\mathrm{s}, l m}^{(p)}$ of the scattered field only, while $F_{\text {ext }}(\hat{\mathbf{u}})$ depends both on $A_{\mathrm{s}, l m}^{(p)}$ and on the amplitudes $W_{\mathrm{i}, l m}^{(p)}$ of the incident field. This dependence is analogous to that on the scattering and extinction cross sections for the force exerted by a plane wave, hence the subscripts [64]:

$$
\mathbf{F}_{\mathrm{rad}}^{\mathrm{PW}}=\frac{n_{\mathrm{m}}}{c} I_{\mathrm{i}}\left[\sigma_{\text {ext }}-g_{\mathrm{i}} \sigma_{\text {scat }}\right] \hat{\mathbf{k}}_{\mathrm{i}},
$$

where the asymmetry parameter in the direction of the incoming wave is

$$
g_{\mathrm{i}}=\frac{1}{\sigma_{\text {scat }}} \oint_{\Omega} \frac{\mathrm{d} \sigma_{\text {scat }}}{\mathrm{d} \Omega} \hat{\mathbf{r}} \cdot \hat{\mathbf{k}}_{\mathrm{i}} \mathrm{d} \Omega .
$$

\subsubsection{Optical torque}

For the calculation of the radiation torque, we start from Eq. (22). By expressing the total fields as $\mathbf{E}_{\mathrm{t}}=\mathbf{E}_{\mathrm{i}}+\mathbf{E}_{\mathrm{s}}$ and $\mathbf{B}_{\mathrm{t}}=\mathbf{B}_{\mathrm{i}}+\mathbf{B}_{\mathrm{s}}$, it is possible to generalise the result originally derived by Marston and Crichton [79] for the torque transferred to an absorbing sphere. In fact, it can be shown [69] that the axial $z$-component of the torque transferred by light along the propagation direction, i.e. $T_{\mathrm{rad}, z}=\mathbf{T}_{\mathrm{rad}} \cdot \hat{\mathbf{z}}$, is given by:

$$
T_{\mathrm{rad}, z}=\underbrace{-\frac{\varepsilon_{\mathrm{m}} E_{\mathrm{i}}^{2}}{2 k_{\mathrm{m}}^{3}} \sum_{p l m} m \operatorname{Re}\left\{W_{\mathrm{i}, l m}^{(p)} A_{\mathrm{s}, l m}^{(p) *}\right\}}_{\text {extinction }}-\underbrace{\frac{\varepsilon_{\mathrm{m}} E_{\mathrm{i}}^{2}}{2 k_{\mathrm{m}}^{3}} \sum_{p l m} m\left|A_{\mathrm{s}, l m}^{(p)}\right|^{2}}_{\text {scattering }},
$$

where we have distinguished the extinction and scattering contributions so that

$$
T_{\mathrm{rad}, z}=T_{\mathrm{ext}, z}-T_{\mathrm{scat}, z} .
$$

The transverse components of the radiation torque, i.e. $T_{\mathrm{rad}, x}=\mathbf{T}_{\mathrm{rad}} \cdot \hat{\mathbf{x}}$ and $T_{\mathrm{rad}, y}=\mathbf{T}_{\mathrm{rad}} \cdot \hat{\mathbf{y}}$, can be calculated in a similar way distinguishing the extinction and scattering contributions [69].

\subsubsection{Amplitudes of a focused beam}

To calculate the multipole amplitudes $\mathcal{W}_{\mathrm{i}, l m}^{(p)}$ of a focused beam, as is used in the case of an optical tweezers, we can exploit the expansion of the incoming beam into plane waves and its focusing in terms of the angular spectrum representation. The detailed description of focal 
fields in the angular spectrum representation is crucial to give an accurate and quantitative modelling of optical tweezers without approximations [80-82].

The expansion of the focused beam around the focal point is given by:

$$
\mathbf{E}_{\mathrm{f}}(x, y, z)=\frac{i k_{\mathrm{t}} f \mathrm{e}^{-i k_{\mathrm{t}} f}}{2 \pi} \int_{0}^{\theta_{\max }} \sin \theta \int_{0}^{2 \pi} \mathbf{E}_{\mathrm{ff}, \mathrm{t}}(\theta, \varphi) \mathrm{e}^{i\left[k_{\mathrm{t}, x} x+k_{\mathrm{t}, y} y\right]} \mathrm{e}^{i k_{\mathrm{t}, z} z} \mathrm{~d} \varphi \mathrm{d} \theta,
$$

where we have taken into account that each plane wave transmitted through the objective lens $\mathbf{E}_{\mathrm{ff}, \mathrm{t}}(\theta, \varphi)$ can be expanded into multipoles:

$$
\mathbf{E}_{\mathrm{ff}, \mathrm{t}}(\theta, \varphi) \equiv \mathbf{E}_{\mathrm{i}}(r, \hat{\mathbf{r}})=E_{\mathrm{i}} \sum_{l m} W_{\mathrm{i}, l m}^{(1)}\left(\hat{\mathbf{k}}_{\mathrm{i}}, \hat{\mathbf{e}}_{\mathrm{i}}\right) \mathbf{J}_{l m}^{(1)}\left(k_{\mathrm{m}} r, \hat{\mathbf{r}}\right)+W_{\mathrm{i}, l m}^{(2)}\left(\hat{\mathbf{k}}_{\mathrm{i}}, \hat{\mathbf{e}}_{\mathrm{i}}\right) \mathbf{J}_{l m}^{(2)}\left(k_{\mathrm{m}} r, \hat{\mathbf{r}}\right),
$$

with the appropriate amplitudes $[67,81,82]$. In the case of a focused field, these amplitudes are

$$
\mathcal{W}_{\mathrm{i}, l m}^{(p)}=\frac{i k_{\mathrm{t}} f \mathrm{e}^{-i k_{\mathrm{t}} f}}{2 \pi} \int_{0}^{\theta_{\max }} \sin \theta \int_{0}^{2 \pi} E_{\mathrm{i}}(\theta, \varphi) W_{\mathrm{i}, l m}^{(p)}\left(\hat{\mathbf{k}}_{\mathrm{i}}, \hat{\mathbf{e}}_{\mathrm{i}}\right) \mathrm{d} \varphi \mathrm{d} \theta .
$$

If the centre around which the expansion is performed is displaced by $\mathbf{P}$ with respect to the focal point $\mathbf{O}$ (inset in Fig. 4), the multipole expansion coefficients can be obtained from Eq. (34), so that we have

$$
\mathcal{W}_{\mathrm{i}, l m}^{(p)}(\mathbf{P})=\frac{i k_{\mathrm{t}} f \mathrm{e}^{-i k_{\mathrm{t}} f}}{2 \pi} \int_{0}^{\theta_{\max }} \sin \theta \int_{0}^{2 \pi} E_{\mathrm{i}}(\theta, \varphi) W_{\mathrm{i}, l m}^{(p)}\left(\hat{\mathbf{k}}_{\mathrm{i}}, \hat{\mathbf{e}}_{\mathrm{i}}\right) \mathrm{e}^{i \mathbf{k}_{\mathrm{t}} \cdot \mathbf{P}} \mathrm{d} \varphi \mathrm{d} \theta .
$$

The amplitudes $\mathcal{W}_{l m}^{(p)}(\mathbf{P})$ define the focal field and can be numerically calculated once the characteristics of the optical system are known.

The radiation force and torque are calculated from knowledge of the scattered amplitudes $\mathcal{A}_{\mathrm{s}, l m}^{(p)}$, e.g. by using the T-matrix [Eq. (25)]. In particular, the radiation force on the particle is given by the expression of the force Eq. (27) by changing $E_{\mathrm{i}} W_{\mathrm{i}, l m}^{(p)} \rightarrow \mathcal{W}_{\mathrm{i}, l m}^{(p)}(\mathbf{P})$ and $E_{\mathrm{i}} A_{\mathrm{s}, l m}^{(p)} \rightarrow \mathcal{A}_{\mathrm{s}, l m}^{(p)}$. Analogous considerations hold true for the calculation of the radiation torque, so that the expressions of the radiation torque by a focused field are obtained by applying the same substitutions to Eq. (32).

\subsubsection{Alternative and hybrid methods}

As a final note, we stress that each method used to calculate optical forces has its own advantages and disadvantages. For example, calculating the T-matrix in optical trapping problems is useful and computationally effective because it is possible to exploit its rotation and translation properties to obtain at once optical forces and torques for different positions and orientations of the trapped particles [65,67,72-75,77]. An equivalent way to use the multipole expansion is given by the so-called generalised Lorenz-Mie theories (GLMTs), where a generic laser beam is expanded in vector spherical harmonics, and the scattering problem is solved for symmetric scatterers, e.g. spheres, so that separation of variables can be used to obtain the expansion coefficients of the scattered fields [83]. The precise connection between the T-matrix formulation and GLMTs can be found in [84], while a description of the use of GLMTs for calculations in optical tweezers can be found, e.g. in [81,85]. 
Other alternative methods rely on the use of the discrete dipole approximation (DDA) and finite-difference time-domain (FDTD) methods. These approaches, although more computationally intensive than the T-matrix, can be readily applied to particles of any shape and composition, and for any light field configuration.

To take advantage of the complementary properties of different methods, hybrid methods have also been developed that make use, e.g. of the T-matrix obtained by point-matching the fields at the particle surface $[86,87]$ or the near-fields calculated with the DDA method $[88,89]$ to get the radiation force and torque on non-spherical scatterers. These mixed approaches are particularly well suited to the calculation of optical forces and torques on optically trapped non-spherical particles and composites. An accurate computational comparison of optical forces on cylinders calculated using the T-matrix formulation with different methods (extended boundary condition, point-matching, and DDA) can be found in [90].

\section{Brownian motion in optical tweezers}

An important aspect of optical trapping and manipulation is the ubiquitous presence of Brownian motion. In fact, microscopic particles undergo a perpetual random motion due to collisions with the molecules of the fluid in which they are immersed. The motion of an optically trapped particle is, therefore, the result of the interplay between this random motion and the deterministic optical forces.

In the early nineteenth century, the botanist Brown [91] gave the first detailed account of Brownian motion. While he was examining aqueous suspensions of pollen grains, he found that microscopic particles contained within these grains were always in rapid oscillatory motion. This movement had been observed previously, but it had been wrongly explained by supposing that these particles were alive. Brown ruled out this possibility with a simple, yet brilliant, experiment: he repeated his observations on some ashes from his chimney, which he could safely assume not to be alive, and the motion was still there.

Brownian motion never ceases even for a system isolated from external perturbations, i.e. it is a phenomenon that happens at thermodynamic equilibrium and is not due to external perturbations. Brownian motion increases as the particle becomes smaller, as the viscosity of the fluid decreases and as the temperature increases, while, at least to a first approximation, it does not depend on the composition and mass of the particle. The resulting Brownian trajectories are very irregular, composed of translations and rotations, to the point that they appear to have no tangent, their velocity is not well-defined and the motion of a particle at one particular instant is independent of the motion of that particle at any other instant.

Even though in principle it would be possible to construct a model of Brownian motion by writing down Newtonian equations of motion for each particle, this is a practically impossible task due to the huge number of molecules in any real situation-a number on the order of the Avogadro number $N_{\mathrm{A}}=6.02 \times 10^{23}$. Thus, to reduce the number of effective degrees of freedom many theories of Brownian motion have been developed during the past century [92]. These theories lie along two main lines:

1. The first approach focuses on the stochastic trajectory $r(t)$ of a single particle, whose motion is modelled with a differential equation to which a stochastic force term is added to account for the interaction of the particle with its environment (Fig. 5a);

2. The second approach focuses on the probability density distribution $\rho(r, t)$ of an ensemble of Brownian particles, whose deterministic evolution is modelled using partial differential equations (Fig. 5b, c). 


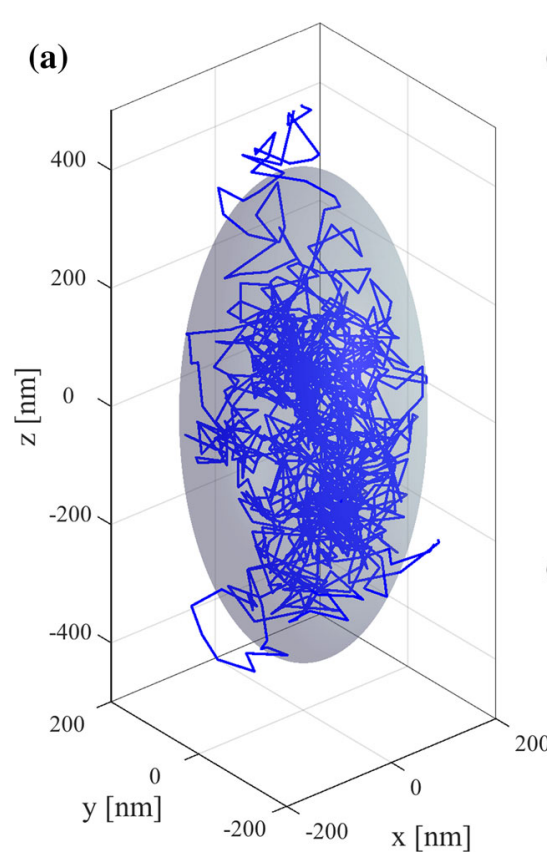

(b)

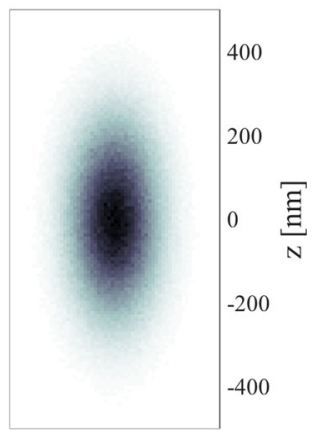

(c)

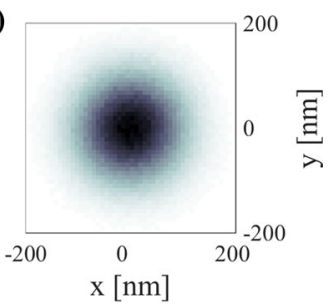

Fig. 5 Simulation of the motion of an optically trapped particle. a Trajectory of a Brownian particle in an optical trap. $\mathbf{b}, \mathbf{c}$ Probability density of finding the particle $\mathbf{b}$ in the $x z$-plane and $\mathbf{c}$ in the $x y$-plane

Not surprisingly, these approaches are strongly connected and, in fact, they can be seen as the two sides of the same coin. On the one hand, probability density distributions can be obtained by averaging over many trajectories and, on the other hand, the statistical properties of the random forces used to calculate the trajectories depend on the probability density distributions $[93,94]$.

\subsection{Random walks}

A random walk is obtained by summing up the terms of a sequence of independent random numbers with any probability distribution. For a sufficiently large number of steps, the resulting random walk has universal properties that do not depend on the details of this probability distribution, at least as long as the random numbers have the same mean and variance.

Brownian motion can be described as such a random walk: when a particle in a fluid receives an impulse due to a collision with a solvent molecule, its velocity changes, but, if the fluid is very viscous, this change is quickly dissipated so that the net result of an impact is a displacement of the particle; this kind of behaviour is typical of systems in the low Reynolds number regime (the Reynolds number, Re, is the ratio of inertial to viscous forces) [95]. The cumulative effect of multiple collisions is to produce the random walk of the particle. If the particle is at position $r_{i}$ at time $t_{i}=i \Delta t$, where $\Delta t$ is the time step, $r_{i}$ evolves according to

$$
r_{i+1}=r_{i}+\xi_{i}
$$

where $\xi_{i}$ is a random displacement whose probability distribution $p_{\xi}(\xi)$ has zero mean and standard deviation $\sigma_{\xi}$ depending on $\Delta t$. 
The precise form of a Brownian motion is obviously not predictable, since it depends on a sequence of random events. However, analysing the Brownian motion of several particles, it is possible to identify some average properties, which are deterministic. For example, the average particle displacement after $h$ time steps is zero because in each time step the displacement has zero mean. However, the mean particle displacement does not deliver a lot of information about the random walk, but there are other, more informative, average properties. In particular, the mean square displacement (MSD) after $h$ time steps quantifies how a particle moves away from its initial position. For example, for ballistic motion, the MSD is proportional to $t^{2}$. The MSD of a Brownian particle, instead, is proportional to $t$, i.e.

$$
\left\langle\Delta r(h)^{2}\right\rangle=\left\langle\left(x_{h}-x_{0}\right)^{2}\right\rangle=\overline{\left(x_{i+h}-x_{i}\right)^{2}}=2 D t,
$$

where $t=h \Delta t, D$ is the diffusion coefficient, the angled brackets denote an ensemble average, i.e. an average over the different Brownian particles, and the overbar denotes a time average, i.e. an average in time of the Brownian motion of one given particle. When dealing with ergodic systems, as is most often the case, the two averages coincide.

In many cases there is also an average drift pushing a Brownian particle during its random walk. The resulting motion is a biased random walk. For example, in the low Reynolds number regime, a uniform external force $F$ pushes a Brownian particle with a constant drift velocity $v_{\text {drift }}=F / \gamma$, where $\gamma$ is the particle friction coefficient, producing a displacement $v_{\text {drift }} \Delta t$ in a time step, so that Eq. (36) becomes

$$
r_{i+1}=r_{i}+v_{\mathrm{drift}} \Delta t+\xi_{i},
$$

where the second and third terms on the right-hand-side of the Eq. (38) are responsible for the drift and the diffusion of the Brownian particle, respectively.

\subsection{The Langevin equation}

By adding a fluctuating force to Newton's equation of motion for a particle of mass $m$ in a viscous fluid one obtains the Langevin equation [96]

$$
m \frac{\mathrm{d}^{2}}{\mathrm{~d} t^{2}} r(t)=-\gamma \frac{\mathrm{d}}{\mathrm{d} t} r(t)+\chi(t)
$$

where $\gamma$ is the particle friction coefficient, which for a spherical particle of radius $a$ moving in a fluid of viscosity $\eta$, is determined by Stokes' law

$$
\gamma=6 \pi \eta a
$$

and $\chi(t)$ is a random force with zero mean, i.e. $\langle\chi(t)\rangle=0$, uncorrelated to the actual particle position, i.e. $\langle\chi(t) x(t)\rangle=0$, and fluctuating much faster than the particle position, i.e. $\langle\chi(t) \chi(t+\tau)\rangle=2 S \delta(\tau)$ where the prefactor $2 S$ is the intensity of the noise. Because of these three properties, $\chi(t)=\sqrt{2 S} W(t)$, where $W(t)$ is a white noise.

In the presence of an external potential $U(r)$, and therefore of a force $F(r)=-\frac{\mathrm{d}}{\mathrm{d} r} U(r)$ acting on the particle, Eq. (39) becomes

$$
m \frac{\mathrm{d}^{2}}{\mathrm{~d} t^{2}} r(t)=-\frac{\mathrm{d}}{\mathrm{d} r} U(r)-\gamma \frac{\mathrm{d}}{\mathrm{d} t} r(t)+\chi(t) .
$$

The fluid damps the colloidal particle motion as in the free-diffusion case, but now the confining potential limits the particle displacement so that the particle explores only a limited region. A particularly important case, which was first studied by [97], is when the potential is harmonic. 
In the low-Reynolds-number regime, it is possible to drop the inertial term in Eq. (41), obtaining the overdamped Langevin equation

$$
\frac{\mathrm{d}}{\mathrm{d} t} r(t)=-\frac{1}{\gamma} \frac{\mathrm{d}}{\mathrm{d} r} U(r)+\xi(t),
$$

where $\xi(t)=\sqrt{2 D} W(t)$ is a white noise with intensity $2 D$, where $D$ is the diffusion coefficient.

The diffusion and friction coefficients, $D$ and $\gamma$, respectively, are closely related to each other and to the average kinetic energy of a particle in a heat bath, i.e. $\frac{1}{2} k_{\mathrm{B}} T$, where $k_{\mathrm{B}}$ is the Boltzmann constant and $T$ is the absolute temperature. In particular, one obtains:

$$
D=\frac{k_{\mathrm{B}} T}{\gamma}
$$

Equation (43) is the simplest statement of the fluctuation-dissipation theorem, which relates the intensity of the fluctuations $(D)$ to the rate of energy dissipation $(\gamma)$ in a system at thermal equilibrium. There are various statements of the fluctuation-dissipation theorem, which apply to different situations. The crucial points to keep in mind are that it applies to systems that are at thermal equilibrium and that it relates the intensity of the thermal noise and the dynamical response of a system [98].

In the presence of a force $F(r)$ and an associated potential $U(r)=-\int F(r) \mathrm{d} r$, using the Maxwell-Boltzmann distribution, the equilibrium probability density is

$$
\rho(r)=\rho_{0} \exp \left(-\frac{U(r)}{k_{\mathrm{B}} T}\right),
$$

where $\rho_{0}=\left[\int \exp \left(-\frac{U(r)}{k_{\mathrm{B}} T}\right) \mathrm{d} r\right]^{-1}$ is the probability density normalisation factor.

Diffusion gradients emerge naturally when a Brownian particle is in a complex or crowded environment. For example, diffusion gets hindered when a particle is close to a wall due to hydrodynamic interactions. These interactions are extremely long-ranged and must therefore be often taken into account. Diffusion gradients are often encountered in the practice of optical manipulation, e.g. when particles are optically trapped near a coverslip or near other particles [99-103]. We will again consider a one-dimensional case, but our conclusions can be straightforwardly generalised to the multidimensional case. We consider the one-dimensional Langevin equation

$$
\frac{\mathrm{d}}{\mathrm{d} r} r(t)=\frac{F(r)}{\gamma(r)}+\sqrt{2 D(r)} W(t)
$$

with a position-dependent diffusion coefficient $D(r) . D(r)$ and $\gamma(r)$ are related by the fluctuation-dissipation relation, which generalises Eq. (43),

$$
D(r)=\frac{k_{\mathrm{B}} T}{\gamma(r)} .
$$

The integration of Eq. (45) presents some difficulties due to the irregularity of the Wiener process [101,102,104-108]. This, in particular, leads to the need of taking into account the presence of a spurious drift, which emerges in the presence of diffusion gradients and is necessary to preserve the relation between the external forces $F(r)$ acting on the particle and the Maxwell-Boltzmann probability distribution $\rho(r)$ given by Eq. (44).

The diffusion constant of a spherical particle of radius $a$ near a flat wall is of particular importance for optical tweezers experiments. Its derivation can be found in [109]. In particular, the diffusion coefficient in the direction parallel to the flat wall can be approximated by 
Faxén formula [110], i.e.

$$
\frac{D_{\|}(h)}{D(\infty)}=1-\frac{9}{16}\left(\frac{a}{h}\right)+\frac{1}{8}\left(\frac{a}{h}\right)^{3}-\frac{45}{256}\left(\frac{a}{h}\right)^{4}-\frac{1}{16}\left(\frac{a}{h}\right)^{5}+\mathcal{O}\left(\left(\frac{a}{h}\right)^{6}\right),
$$

where $D(\infty)$ is the bulk diffusion coefficient and $h$ is the distance between the centre of the particle and the flat wall. There is no second-order term in the denominator, so this formula remains good to within $1 \%$ for $h>3 a$ if one ignores all but the first-order term, i.e.

$$
\frac{D_{\|}(h)}{D(\infty)} \approx 1-\frac{9}{16} \frac{a}{h} .
$$

The diffusion coefficient in the vertical direction has a more complicated form, but can approximated as well to first order obtaining:

$$
\frac{D_{\perp}(h)}{D(\infty)} \approx 1-\frac{9}{8} \frac{a}{h} .
$$

\subsection{Brownian dynamics simulations}

The presence of the stochastic term $\xi(t)$ makes the integration of the Langevin equation difficult because advanced mathematical tools are required. In the following, we will show how to integrate stochastic equations using a simple finite difference algorithm [111]. The solution of an ordinary differential equation using this algorithm is straightforward. Considering regular time steps $t_{i}=i \Delta t$, the finite difference equation corresponding to each time step has a solution $x_{i}$. If $\Delta t$ is sufficiently small, then $x_{i} \approx x\left(t_{i}\right)$. Therefore the continuous solution $x(t)$ is approximated by the sequence of discrete values. The finite difference equation is obtained from the ordinary differential equation by replacing: $x(t)$ by $x_{i}$, the first derivative term $\frac{\mathrm{d} x(t)}{\mathrm{d} t}$ by $\left(x_{i}-x_{i-1}\right) / \Delta t$ and the second derivative term $\frac{\mathrm{d}^{2} x(t)}{\mathrm{d} t^{2}}$ by $\left(x_{i}-2 x_{i-1}+x_{i-2}\right) / \Delta t^{2}$. Once the finite difference equation is solved for $x_{i}$, the solution is obtained recursively using the values of the previous iterations.

The noise term, i.e. $\chi(t)=\sqrt{2 S} W(t)$ or $\xi(t)=\sqrt{2 D} W(t)$, cannot be approximated by its instantaneous values at times $t_{i}$, because these values are not well-defined (due to the lack of continuity), and their magnitude varies wildly (due to the infinite variation). To treat a white noise $W(t)$ within a finite difference approach a discrete sequence of random numbers $W_{i}$ that mimics the properties of $W(t)$ is needed. This can be achieved by a sequence of uncorrelated random numbers with zero mean and variance $1 / \Delta t$. Practically a sequence $w_{i}$ of Gaussian random numbers with zero mean and unit variance is generated then rescaled to obtain the sequence $W_{i}=w_{i} / \sqrt{\Delta t}$ which has variance $1 / \Delta t$. The time step $\Delta t$ should be much smaller than the characteristic time scales of the stochastic process to be simulated. If $\Delta t$ is comparable to or larger than the smallest time scale, the numerical solution typically will not converge to the correct solution and may show an unphysical oscillatory or diverging behaviour.

A Brownian particle in an optical trap is in a dynamic equilibrium where the thermal noise tries to push it out of the trap and optical forces drive it towards the potential energy minimum. The time scale on which the restoring force acts is given by the ratio $\tau_{\mathrm{ot}}=\gamma / \kappa$. Typically, $\tau_{\mathrm{ot}}$ is significantly longer than the momentum relaxation time $\tau_{\mathrm{m}}=\mathrm{m} / \gamma$, which is very short, typically on the order of a fraction of a microsecond for the case of a $1 \mu \mathrm{m}$ diameter silica bead. Therefore, it is possible to ignore inertial effects and use an overdamped equation such as Eq. (42), where the only relevant time scale is $\tau_{\mathrm{ot}}$. This approach has the advantage that one can employ a relatively large time step, i.e. $\Delta t \gg \tau_{\mathrm{m}}$. The time step $\Delta t$ should, however, still be significantly smaller than $\tau_{\mathrm{ot}}$ for the reasons discussed above. 
For a three-dimensional optical trap one can employ a set of three independent overdamped Langevin equations [Eq. (42)] with a harmonic restoring force:

$$
\left\{\begin{array}{l}
\frac{\mathrm{d} x(t)}{\mathrm{d} t}=-\frac{\kappa_{x}}{\gamma} x(t)+\sqrt{2 D} W_{x}(t) \\
\frac{\mathrm{d} y(t)}{\mathrm{d} t}=-\frac{\kappa_{y}}{\gamma} y(t)+\sqrt{2 D} W_{y}(t) \\
\frac{\mathrm{d} z(t)}{\mathrm{d} t}=-\frac{\kappa_{z}}{\gamma} z(t)+\sqrt{2 D} W_{z}(t)
\end{array}\right.
$$

where $x$ and $y$ represent the position of the particle in the plane perpendicular to the beam propagation direction and $z$ represents the position of the particle along the propagation direction. The stiffnesses of the trap in each of these directions are $\kappa_{x}, \kappa_{y}$ and $\kappa_{z}$ respectively, $\gamma$ is the particle friction coefficient and $W_{x}(t), W_{y}(t)$ and $W_{z}(t)$ are independent white noises. The corresponding system of finite difference equations is

$$
\left\{\begin{array}{l}
x_{i}=x_{i-1}-\frac{\kappa_{x}}{\gamma} x_{i-1} \Delta t+\sqrt{2 D \Delta t} w_{x, i} \\
y_{i}=y_{i-1}-\frac{\kappa_{y}}{\gamma} y_{i-1} \Delta t+\sqrt{2 D \Delta t} w_{y, i} \\
z_{i}=z_{i-1}-\frac{\kappa_{z}}{\gamma} z_{i-1} \Delta t+\sqrt{2 D \Delta t} w_{z, i}
\end{array}\right.
$$

where $x_{i}, y_{i}$ and $z_{i}$ represent the position of the particle at time $t_{i}$, and $w_{i, x}, w_{i, y}$ and $w_{i, z}$ are independent Gaussian random numbers with zero mean and unitary variance.

The line in Fig. 5a shows a simulated trajectory of a Brownian particle in an optical trap with $\kappa_{x}=\kappa_{y}=1.0 \mathrm{fN} / \mathrm{nm}$ and $\kappa_{z}=0.2 \mathrm{fN} / \mathrm{nm}$. The fact that the trapping stiffness along the beam propagation axis $(z)$ is smaller than in the perpendicular plane $(x y)$ is commonly observed in experiments and is mainly due to the different intensity distribution along the different axes and to the presence of scattering forces along the optical axis. Thus, the particle explores an ellipsoidal volume around the centre of the trap, represented in Fig. 5a by the shaded grey equiprobability surface. In Fig. 5b, c, we show the projections of the probability density of finding the particle onto the $x z$ - and $x y$-planes, respectively.

The time scale $\tau_{\mathrm{ot}}$, which characterises how quickly a particle relaxes towards equilibrium, can be seen in the position autocorrelation function (ACF):

$$
\mathcal{C}_{x}(\tau)=\overline{x(t+\tau) x(t)} .
$$

As the stiffness increases, the particle undergoes a stronger restoring force and the correlation time decreases, because the particle explores a smaller phase-space. Unlike the free diffusion case [Eq. (37)], the MSD

$$
\left\langle\Delta x(\tau)^{2}\right\rangle=\overline{[x(t+\tau)-x(t)]^{2}}
$$

does not increase indefinitely, but reaches a plateau because of the confinement imposed by the trap. The transition from the linear growth (corresponding to the free diffusion behaviour) to the plateau (due to the confinement) occurs at about $\tau_{\mathrm{ot}}$.

\section{Experimental setups}

Even though the basic principle of optical tweezers requires the use of a single strongly focused laser beam to trap and manipulate a microscopic particle, ever more complex exper- 
(a)
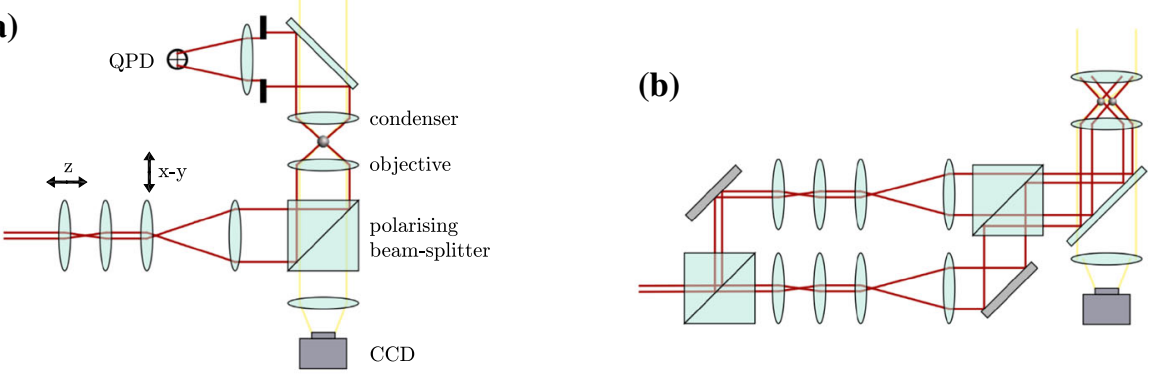

(c)

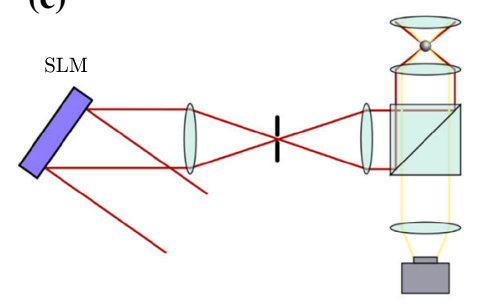

(d)

(e)

(f)
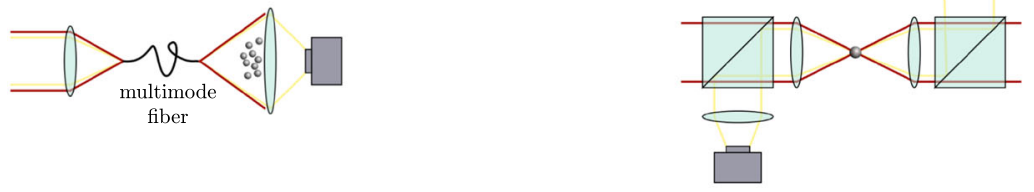

(g)

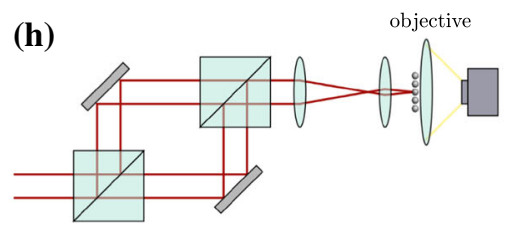

Fig. 6 Typical optical tweezers setups: a single-beam optical tweezers with quadrant photodiode, $\mathbf{b}$ doublebeam optical tweezers with mechanically steerable trapping beams, $\mathbf{c}$ holographic optical tweezers incorporating a spatial light modulator (SLM), d time-sharing optical tweezers using an acousto-optic deflector (AOD), e speckle optical tweezers generating the speckle light field using a multimode fibre, $\mathbf{f}$ counter-propagatingbeam optical tweezers, $\mathbf{g}$ optical stretcher, $\mathbf{h}$ interferometric optical tweezers to generate large-scale optical potentials

imental setups have been developed to perform novel and challenging experiments. Some examples of different trapping schemes are depicted in Fig. 6 and some examples of optically manipulated particles are shown in Fig. 7. Some detailed instruction on how to build advanced optical tweezers setups are provided in [11,112]; other useful references are [113-118]. In the following, we review the main building blocks and techniques to construct and calibrate optical tweezers systems. 

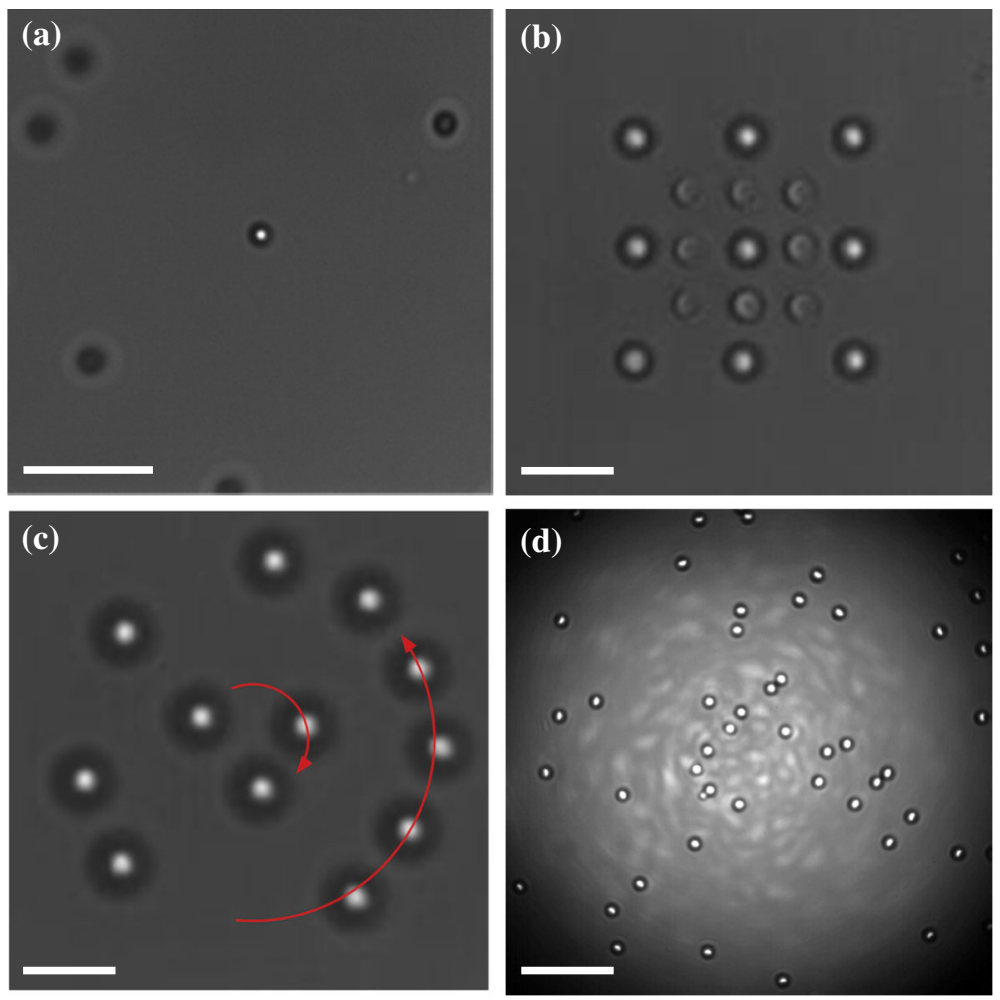

Fig. 7 Optical manipulation examples: a a particle trapped in a single-beam optical tweezers, b 18 particles held in a multi-trap holographic optical tweezers based on the use of a spatial light modulator, c several particles set in rotation by the transfer of orbital angular momentum from a high order Laguerre-Gaussian beam generated by means of a spatial light modulator, $\mathbf{d}$ particles optically trapped using a speckle light field. Figures adapted from [112]

\subsection{Microscopes}

The most convenient choice to built an optical tweezers setup is to use a conventional commercial light microscope. This choice has many advantages but also some drawbacks. Commercial microscopes can be modified to host a dichroic mirror before the objective lens to deflect the trapping laser beam into the lens and at the same time to transmit the illumination light to a camera to image the sample. They are easy to use and are optimised to reduce any aberration or distortion of the sample images, but they may be relatively difficult to customise, for example, to add a position sensitive device. Moreover, the optics are usually optimised for visible light and therefore some issues arise when infrared laser sources are used. Finally, commercial microscopes do not offer the level of mechanical stability required for the most sensitive nanometer-scale experiments. Despite the difficulties in the design and construction of a home-made microscope, choosing this option is increasingly popular. These microscopes can be built directly with standard optomechanical components or with the use of a user-designed frame. In these microscopes the access to any part is very easy and with the proper choice of materials and design the mechanical stability is extremely high. It 
is also worth noting that home-made microscopes are often significantly less expensive than commercial ones.

\subsection{Laser sources}

The quality of the laser beam is critical to achieve the tightly focused spot required for optical trapping, which must be as close to the diffraction limit as possible. The quality of the laser beam is often expressed using the parameter $M^{2}$, which is the ratio between the beam parameter product of the laser beam and that of a diffraction-limited beam $[119,120]$. A Gaussian beam has $M^{2}=1$ and, for optical tweezers applications, a laser beam with $M^{2}$ as close as possible to this diffraction limited performance is preferable. Good pointing stability is required to maintain the position of the optical trap steady. Fluctuations in beam pointing direction can arise from, e.g. mechanical vibrations of optical elements in the laser resonator or thermal effects in the laser gain medium. Several different quantities are used to express the pointing stability, so care should be exercised when trying to interpret this parameter, paying attention in particular to the conditions under which it has been measured and to whether the quoted stability refers to the average direction of the beam or to the root-mean-square (rms) fluctuations in direction. In optical trapping experiments, fluctuations in laser power lead to fluctuations in the strength of the optical trap. In laser data sheets fluctuations in laser power are usually quantified by the power stability, i.e. the drift in the average laser power measured over an extended period of time, and the noise, i.e. fluctuations around the average of the laser power within a specified bandwidth, typically from a few hertz to (tens of) megahertz. It is worth mentioning that laser sources based on a monolithic non-planar ring oscillator Nd:YAG crystal exhibit optical properties unmatched by any other product, such as an output tunable over $30 \mathrm{GHz}$ with the extraordinarily narrow linewidth of about $1 \mathrm{kHz}$ and extremely low noise. These sources are perfect for experiments in which very weak forces $(\approx 1 \div 10 \mathrm{fN})$ are involved.

\subsection{Particle tracking}

Particle tracking is the key technique for quantitative measurements with optical tweezers. Most measurements that can be done with an optical tweezers setup are based on the knowledge of the particle position. There are two possibilities for measuring the particle position: the first is to image the trapped particle using a CCD or CMOS camera, while the second is to use detectors capable of measuring the spatial distribution of intensity in the interference pattern that occurs between the light scattered by the trapped particle and the unscattered laser light.

Since a typical optical tweezers setup comes already equipped with a digital camera, the most straightforward means to measure the motion of a Brownian particle is to record a video of its position and then to track the position of the particle frame by frame. This technique, known as digital video microscopy [121,122], has found widespread application in several fields and, in particular, in colloidal studies. It is especially well-suited to study systems where multiple particles are present, but it is relatively slow being limited by the camera frame rate, which typically goes up to a few thousands frames per second.

Given a greyscale image of the particles to be tracked, the simplest method to track the positions of the particles is by thresholding. Assuming the particle to be lighter than the background, the pixel value at the particle is larger than that of the background, and it is possible to fix a threshold and convert the greyscale image into a black and white image such that pixels whose values are smaller than the threshold are set to black and pixels whose 
value is larger than the threshold are set to white. Following the thresholding operation, it is possible to apply some morphological filters, such as dilation and erosion filters, to eliminate some common causes of noise such as salt-and-pepper noise, i.e. sparsely occurring white and black pixels. The position of each particle can then be calculated as the centroids of the remaining white regions. Thanks to the averaging in the centroid calculation, this technique permits sub-pixel resolution to be achieved, typically down to about a tenth of the pixel size (about $10 \mathrm{~nm}$ ) in the $x$-and $y$-directions. The area of the regions can further be used to estimate the radius of the corresponding particle.

A more advanced particle detection technique, known as feature point detection, makes use of the fact that the particle intensity profiles on the image are, to a first approximation, Gaussian. To achieve the best results, independently of the detection technique employed, often some preliminary steps are required to prepare the images such as elimination of salt-and-pepper noise by median filtering, subtraction of a (fixed) background image and normalisation of the image intensity. It is also important to optimise the illumination. Moreover, using a coherent source of illumination (holographic video microscopy), it is possible to achieve better contrast, especially along the $z$-direction. Furthermore, it has been recently demonstrated that machine learning techniques can significantly improve the performance of digital video microscopy especially with low signal-to-noise ratios [123].

As particle detection is applied to each frame, it delivers a series of sets of positions, each corresponding to the particles detected in the frame acquired at time $t$. At this point, it is necessary to link positions corresponding to the same physical particle in subsequent frames into trajectories. The basic idea of the linking algorithm is that a particle at time $t$ is identified with a particle at time $t+\Delta t$, where $\Delta t$ is the time difference between the two frames, if the two measured positions are less than a certain value. In the case of freely diffusing Brownian particles, this value can be set to a multiple of $\sqrt{4 D \Delta t}$ in order to account for the Brownian diffusion of the particle frame-to-frame. This algorithm can be extended so that each linking step may consider several frames to account for particle occlusion. By performing this linking it is finally possible to obtain the particle trajectories, which can be then used for various kinds of statistical analysis (Fig. 8).

Finally, it is important to be able to convert the particle position measurements expressed in pixels into actual physical units of length. This requires a calibration of the microscope. The easiest way of doing this is by imaging a regular object, e.g. a microfabricated grating. Such gratings can be produced by standard lithography techniques, but it is also possible to acquire them commercially. An alternative approach is to track a particle stuck on the bottom of the sample as this is controllably moved by the stage.

An alternative to digital video microscopy is the use of the interference pattern arising from the interference between the incoming and scattered fields [124]. The condenser collects such a pattern and a photodetector located on the condenser back-focal plane records the resulting signals. Thus, by tracking the movement of the intensity distribution of the interference pattern, it is possible to measure the particle position in the transverse $x y$-plane. The two crucial parameters of any position detection system are: (1) the displacement sensitivity, i.e. the signal as a function of the particle displacement, typically expressed in volts per metre; and (2) the linear response range of the position detection system. Both parameters depend on the intensity distribution that reaches the detector. The detection bandwidth is also important in the position detection. Some experiments require high bandwidth $\left(f_{\text {acq }} \sim 10^{4} \div 10^{5} \mathrm{~Hz}\right.$ ), as, for instance, the study of non-diffusive Brownian motion at very short times [125-127], the observation of ballistic motion of a particle in a liquid [125,126], and measurement of instantaneous velocity of a particle in vacuum [128]. The majority of the experiments can be performed with detection bandwidth in the range $f_{\text {acq }} \sim 10^{1} \div 10^{3} \mathrm{~Hz}$. This range is 


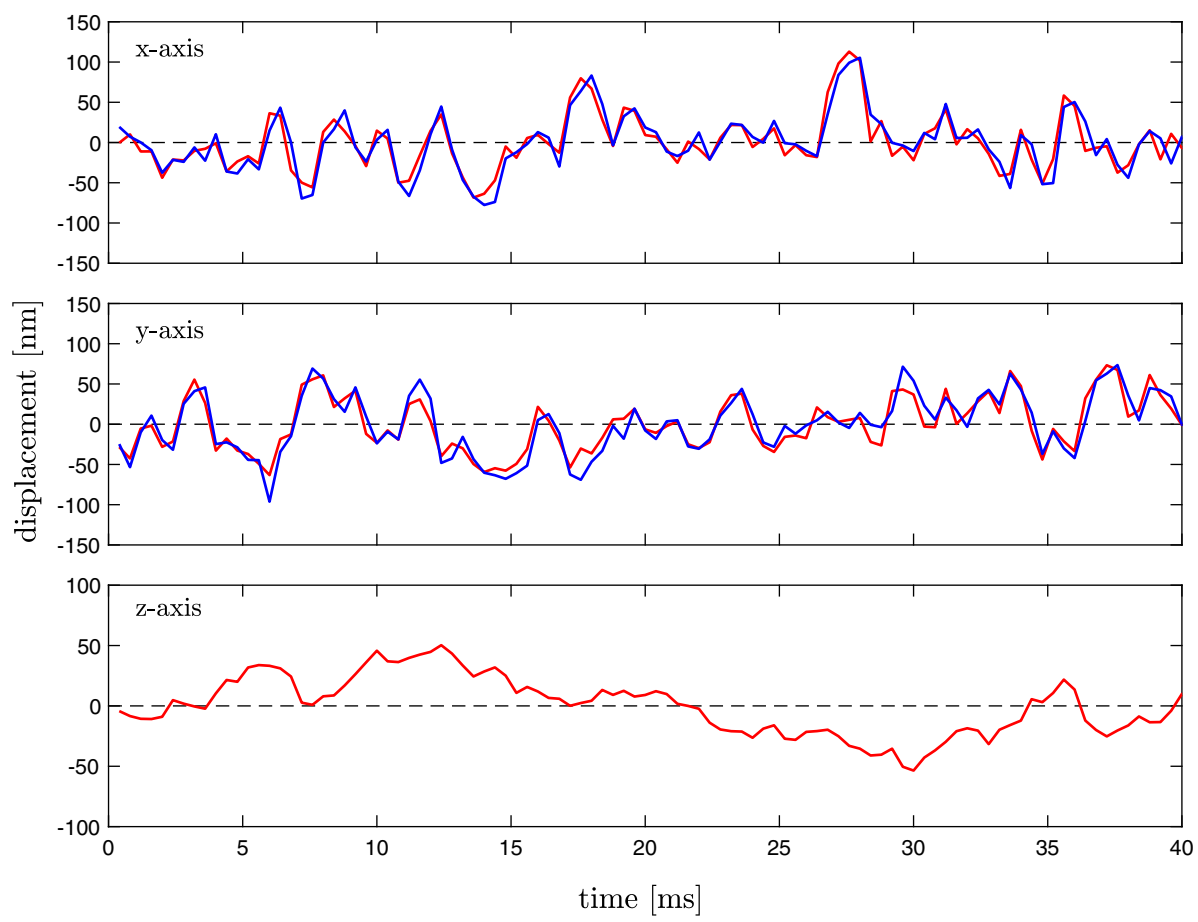

Fig. 8 Tracking of an optically trapped particle. The position of the particle can be tracked using digital video microscopy or interferometry. We obtain an excellent agreement between the positions measured with the two techniques in the $x y$-plane: the blue lines representing the coordinates of the particle measured using digital video microscopy overlap with the red lines representing the coordinates obtained from interferometry. Interferometry also permits the measurement of the vertical $z$-coordinate of the particle

easily accessible by using quadrant photodiodes [129] as well as with modern digital CMOS cameras capable to record frames up to $10000 \mathrm{fps}$. Very high bandwidth, above $10^{6} \mathrm{~Hz}$ can be reached using a special configuration where the forward interference pattern is split in two halves and each of them is sent to a very fast photodiode device, even though this setup limits the detection of movement to only one direction [130]. The trapping and detection operations can be made independent by illuminating the particle with an auxiliary beam weak enough not to generate significant optical forces. This is particularly useful in experiments where the position or intensity of the trapping beam need to be changed during the experiment.

Two types of photodetectors are typically used as position sensors. The quadrant photodiode (QPD) works by measuring the intensity difference between the left-right and top-bottom sides of the detection plane. The position sensing detector (PSD) measures the position of the centroid of the collected intensity distribution, giving a more adequate response for nonGaussian profiles. Both QPD and PSD fare well when assessed against sensitivity and linear range along the transverse direction. This is generally true for the forward scattering detection scheme, but usually it is not true when non-Gaussian intensity profiles are considered, e.g. in the case of backward scattering position detection [131].

When the particles are displaced along the longitudinal $z$-direction, the size of the spot changes as a consequence of the change of relative phase between incoming and scattered wave due to the Gouy phase shift inherent in focused beams [132]. It is convenient to reduce 
the numerical aperture of the detection system to have a linear response around the equilibrium position [133]. The numerical aperture of the detection system can be changed by changing the condenser lens, but also by placing a suitably-sized iris in front of the photodetector.

The forward scattering position detection scheme is not always possible. In a number of experiments, geometrical constraints may prevent access to the forward scattered light, forcing one to make use of the backward scattered light instead. This may occur, for example, in biophysical applications where one of the two faces of a sample holder needs to be coated with some specific non-transparent material, or in plasmonics applications where a plasmon wave needs to be excited from one of the faces of the holder. Furthermore, the backward mode of operation makes it easier to combine the optical trap with other techniques such as atomic force microscopy, which require access to one side of the holder [134-136]. Nevertheless it presents a number of difficulties that are absent from the forward scattering detection [131]: particular attention should be paid to the probe size, as there exist specific sizes for which the probe displacement cannot be detected. Some of these problems may be mitigated using the appropriate detection numerical aperture and choosing either QPD or PSD [131].

Finally, we should remark that all signals obtained with interferometric techniques are not in physical units of length, but typically in volts. Therefore, a calibration of the volts-tolength conversion coefficient needs to be performed. This calibration factor can be obtained via several different techniques [137-141]. In particular, it is possible to scan an optically trapped particle across a (much weaker) detection beam, in which case one need to have at least two beams. Fortunately, it is also possible to perform this calibration self-consistently using only the position signal of an optically trapped particle acquired by a photodetector provided the viscosity of the liquid is known.

\subsection{Calibration techniques}

Once the trajectory of a Brownian particle has been measured either by digital video microscopy or by interferometry, it is possible to use it to study quantitatively the optical potential [11]. In calibrating an optical trap, the main objective is to determine the trap stiffness $\kappa_{x}$ and the conversion factor $S_{x}$ from measurement units (e.g. pixels or volts) to physical units of length (metres). Most commonly, passive calibration techniques are employed, where the trajectory of the optically trapped particle is measured within a fixed optical trap. These techniques include potential analysis [142], the equipartition method, the mean square displacement analysis, the autocorrelation function analysis [114,143-145], the power spectrum analysis [139] and the maximum likelihood estimation analysis [146]. There are also some active calibration techniques, where the effect on the optically trapped particle of a known force is measured, typically by applying a fluid flow. All techniques should deliver the same result within the experimental error; in fact, employing more than one calibration technique and confirming their consistency is a good check of the quality of the acquired experimental data, as shown in Fig. 9 for the three most commonly employed methods. An additional check is given by the fact that the trap stiffness should be proportional to the optical power $[112,147]$.

\subsubsection{Potential analysis}

The trajectory $x(t)$ of a Brownian particle can be described by the Langevin equation (42). Since the Brownian particle is in thermal equilibrium with the heat-bath constituted of the fluid molecules, its probability distribution follows the Maxwell-Boltzmann distribution given by Eq. (44), i.e. 
(a)

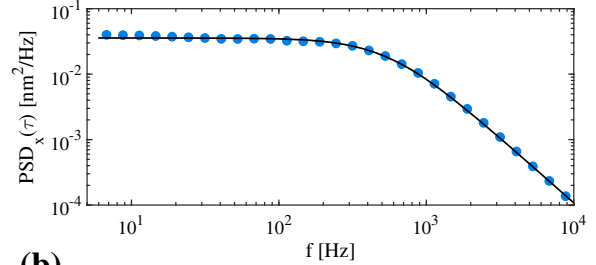

(b)
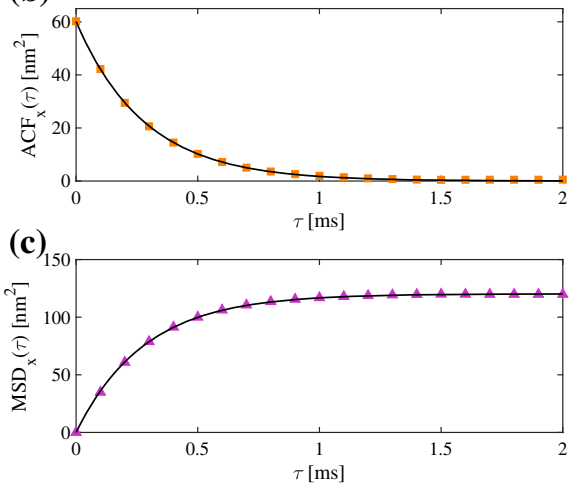

(d)

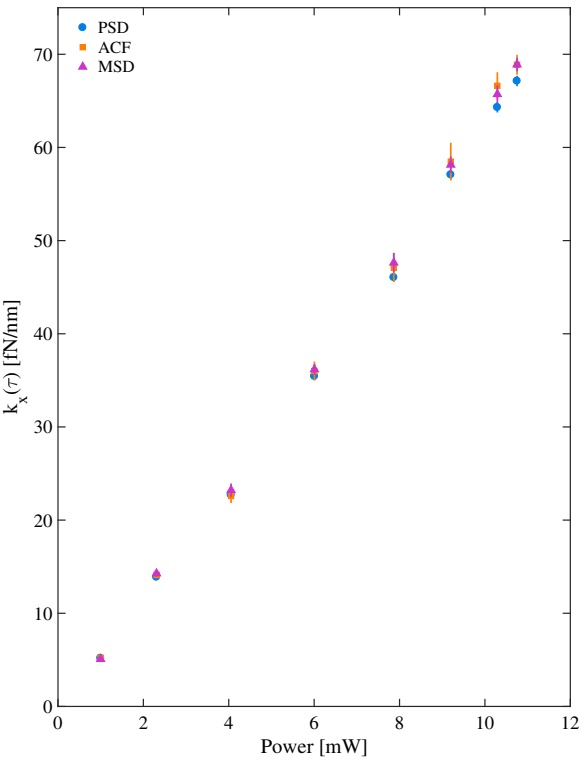

Fig. 9 Calibration of an optical tweezers with the most three common methods. The symbols in a, b and c represent experimental data for the mean square displacement, the autocorrelation function and the power spectral density, while the black solid lines are the corresponding fitting results. $\mathbf{d}$ Stiffnesses as function of the laser power obtained from the fitting procedure. The agreement among the three methods is excellent

$$
\rho(x)=\rho_{0} \exp \left[-\frac{U(x)}{k_{\mathrm{B}} T}\right],
$$

where $\rho_{0}$ is a normalisation factor. By solving Eq. (52) for $U(x)$, we obtain

$$
U(x)=-k_{\mathrm{B}} T \log [\rho(x)]+U_{0},
$$

where $U_{0}$ is a constant. Differently from the methods presented in the following, this method does not assume that the optical tweezers potential is harmonic and, therefore, can be used to verify the hypothesis that the optical potential is harmonic, or to probe more complex potentials.

The determination of the potential with this method is subject to some systematic errors, as the potential can be smeared out by the presence of uncorrelated noise, e.g. low-frequency mechanical vibrations of the setup and detection errors [142]. Also, if the acquired data are correlated, the presence of low-pass frequency filters in the acquisition system can alter the appearance of the potential [148].

\subsubsection{Equipartition method}

The potential associated with an optical trap is to a very good first approximation harmonic, i.e.

$$
U(x)=\frac{1}{2} \kappa_{x}\left[x-x_{\mathrm{eq}}\right]^{2},
$$

where $\kappa_{x}$ is the trap stiffness and $x_{\mathrm{eq}}$ is the equilibrium position. Once this hypothesis has been verified following the procedure described above, it is possible to use the equipartition 
theorem, which states that

$$
\langle U(x)\rangle=\frac{1}{2} \kappa_{x}\left\langle\left(x-x_{\mathrm{eq}}\right)^{2}\right\rangle=\frac{1}{2} k_{\mathrm{B}} T,
$$

where instead of the ensemble average one can also employ a time average because of the ergodicity of the system.

As in the determination of the potential described above, the experimental data points need to sample the probability distribution so that they do not need to be acquired with a fixed time step as long as a sufficient number of independent points is acquired. The estimation of $\kappa_{x}^{(\mathrm{ex})}$ with the equipartition method is also prone to systematic errors due to the presence of uncorrelated noise [142].

\subsubsection{Mean square displacement analysis}

A more precise characterisation of the optical trap can be obtained from the mean square displacement (MSD) of the optically trapped particle (Fig. 9a). The MSD quantifies how a particle moves from its initial position: for ballistic motion, the MSD is proportional to $t^{2}$, for diffusive motion it is proportional to $t$, and for a trapped particle it saturates to a constant value. For the case of an optically trapped particle the MSD is given by

$$
\operatorname{MSD}_{x}(\tau)=\overline{[x(t+\tau)-x(t)]^{2}}=2 \frac{k_{\mathrm{B}} T}{\kappa_{x}}\left[1-\mathrm{e}^{-\frac{|\tau|}{\tau_{\mathrm{to}, x}}}\right],
$$

where $\kappa_{x}$ is the trap stiffness and $\tau_{\text {to }, x}=\gamma / \kappa_{x}$ is the trap characteristic time. The $\operatorname{MSD}_{x}(\tau)$ features a transition from a linear growth corresponding to a free diffusion behaviour at short time scales $\left(\tau \ll \tau_{\mathrm{ot}, x}\right)$ to a plateau due to the confinement al long time scales $\left(\tau \gg \tau_{\mathrm{ot}, x}\right)$.

The experimental MSD can be fitted by a least squares method to the theoretical MSD given by Eq. (56) to obtain $\kappa_{x}^{(\mathrm{ex})}$. By repeating this analysis on various experimental series, it is possible to obtain an average value and an uncertainty for $\kappa_{x}^{(\mathrm{ex})}$, as well as for the $\mathrm{MSD}_{x, k}$.

Often the trajectory obtained from the position detection system is not naturally given in units of length. For example, in the case of digital video microscopy the trajectory is naturally given in pixels and in the case of interferometric detection in voltage. Therefore, it can be useful to determine the conversion factors to units of length by fitting also $\gamma^{(\mathrm{ex})}$. Then, the conversion factor to units of length is given by

$$
S_{x}^{(\mathrm{ex})}=\sqrt{\frac{\gamma}{\gamma^{(\mathrm{ex})}}},
$$

where $\gamma$ is the friction coefficient given by Stokes' Law. This procedure can serve also as a consistency check for the analysis procedure.

\subsubsection{Autocorrelation analysis}

Another method often employed in characterising an optical tweezers is the analysis of the autocorrelation function (ACF) of the particle position [114,143,144] (Fig. 9b). This method is particularly suited when one wishes to deconvolve a more complex time-domain dynamics of the trapped particle from its tracking signal, e.g. in the case of non-conservative effects $[145,149]$ and dynamics of non-spherical particles. The position ACF provides a measure of the time it takes for the particle to "forget" its earlier position. The time scale $\tau_{\mathrm{to}, x}$ characterises 
how a particle falls into the trap: as the stiffness increases, the particle undergoes a stronger restoring force and $\tau_{\mathrm{to}, x}$ decreases. The position $\mathrm{ACF}$ is given by

$$
\mathcal{C}_{x}(\tau)=\frac{k_{\mathrm{B}} T}{\kappa_{x}} \mathrm{e}^{-\frac{|\tau|}{\tau_{\mathrm{to}, x}}},
$$

where $\kappa_{x}$ is the trap stiffness and $\tau_{\mathrm{to}, x}=\gamma / \kappa_{x}$ is the trap characteristic time. The experimental ACF can be fitted by a least squares method to the theoretical ACF given by Eq. (58) to obtain an estimation of $\kappa_{x}$. By repeating this analysis on several experimental series it is possible to obtain an average value and an uncertainty for $\kappa_{x}^{(\mathrm{ex})}$, as well as for the $C_{x, k}$. Also in the case of the ACF analysis, it can be useful to determine the conversion factor from the acquisition system units to units of length by fitting also $\gamma^{(\mathrm{ex})}$ and using Eq. (61).

\subsubsection{Power spectrum analysis}

We now consider the power spectrum analysis of the trajectory of an optically trapped particle (Fig. 9c). The power spectrum analysis is widely regarded as the most reliable method, at least when dealing with spherical particles [139]. In fact, it has the advantage of working in the frequency domain, which permits one to relatively easily remove common causes of noise such as slow mechanical drifts at low frequency and periodic noise, e.g. that due to the mains electrical network, which appears as peaks in the power spectral density (PSD).

Starting from the overdamped Langevin equation of motion of an optically trapped particle given in Eq. (48), one can easily obtain the PSD of the particle motion [139]:

$$
P_{x}(f)=|\check{X}(f)|^{2}=\frac{D /\left(2 \pi^{2}\right)}{f_{\mathrm{c}, x}^{2}+f^{2}},
$$

where we have introduced the corner frequency

$$
f_{\mathrm{c}, x}=\frac{\kappa_{x}}{2 \pi \gamma} .
$$

To perform a least square fitting of the experimentally obtained power spectrum to its theoretical expression [Eq. (59)], each data point need be drawn from a Gaussian distribution and different data points need to be statistically independent. To satisfy these conditions the solution is to perform data compression which results in a smaller data set with less noise and, because of the central limit theorem, in normally distributed data. Data compression can be performed by repeating the experiment $n_{\mathrm{r}}$ times and averaging the resulting values of the experimental power spectrum. Alternatively, it is also possible to perform data compression by blocking. Blocking replaces a block of $n_{\mathrm{b}}$ consecutive data points with a single new data point with coordinates that are simply block averages. When $n_{\mathrm{r}}$ and/or $n_{\mathrm{b}}$ are sufficiently large, the resulting data points have a Gaussian distribution by virtue of the central limit theorem and are, thus, amenable to least square fitting. It is, therefore, possible to fit the value of $f_{\mathrm{c}, x}$. By repeating this analysis on various experimental data series, it is possible to obtain an average value and an uncertainty for $f_{\mathrm{c}, x}$, as well as for the experimental power spectrum.

Also in the case of the PSD analysis, it can be useful to determine the conversion factor from the acquisition system units to units of length. In the case of the PSD analysis, this is most naturally done by fitting also $D^{(\mathrm{ex})}$ and by using the following expression of the conversion factor:

$$
S_{x}^{(\mathrm{ex})}=\sqrt{\frac{D^{(\mathrm{ex})}}{D}},
$$


where $D$ is the diffusion coefficient given by Eq. (43).

The PSD can be effectively used to detect and quantify the presence of noise in the setup. In particular, it is possible to perform two kinds of basic tests. It is possible to record the dark spectrum, i.e. the PSD signal generated when the photodetector is kept in total darkness. This spectrum is a measurement of the equipment's electronic noise. It is also possible to record a light spectrum, i.e. the PSD recorded with the laser light impinging directly onto the photodiode with no microsphere in the trap. This spectrum is a measurement of the setup stability. Compared with the dark spectrum, the light spectrum shows significant lowfrequency noise, mainly due to the mechanical instabilities of the setup. The difference is caused by the limited pointing stability of the unscattered laser beam and of the optics it passes through relative to the photodiode. The light spectrum can be effectively used to identify and eliminate some common causes of mechanical vibrations in the setup, usually due to the presence of some underdamped mechanical resonances.

\subsubsection{Maximum likelihood estimation analysis}

The maximum likelihood estimation analysis builds on the observation that the friction force acting on an optically trapped particle can be written as

$$
f_{n}=\gamma \frac{\Delta x_{n}}{\Delta t_{n}} \approx-k x_{n}+\sqrt{\frac{2 k_{\mathrm{B}} T \gamma}{\Delta t_{n}}} w_{n},
$$

where $\Delta t_{n}$ is a time interval during which the particle moves by $\Delta x_{n}$ from position $x_{n}$. The central observation is that this equation is in the form of a linear regression model, whose parameters can therefore be optimally estimated with a maximum likelihood estimator from a series of observations of the dependent and independent variables [146]. The estimation of the trap stiffness is then

$$
k^{*}=\frac{\sum_{n} x_{n} f_{n}}{\gamma \sum_{n} x_{n}^{2}},
$$

which is a very simple expression that can be computed extremely fast. The residual error is $\sigma^{2}=\frac{1}{N} \sum_{n}\left[f_{n}+k^{*} x_{n}\right]^{2}$; using the fact that $f_{n}+k^{*} x_{n}=\sqrt{\frac{2 k_{\mathrm{B}} T \gamma}{\Delta t_{n}}}$ (Eq. 62), we can estimate the diffusion coefficient as

$$
D^{*}=\frac{1}{N} \sum_{n} \frac{\Delta t_{n}}{2 \gamma}\left[f_{n}+k^{*} x_{n}\right]^{2}
$$

and compare it to the expected value $D$, which provides an intrinsic consistency check for the quality of the result.

\subsubsection{Drag force method}

Until now, we have only considered passive calibration methods. However, it is also possible to infer the spring constant $\kappa_{x}$ by measuring the displacement of the particle in the optical trap resulting from the application of a known force [129]. In the case of a spherical bead surrounded by a fluid medium such as water, this can be accomplished by monitoring the position of the trapped bead while the fluid flows past it at a known velocity $v_{\text {fluid. }}$. The force on the bead due to the fluid flow is then given by

$$
F_{\text {drag }}=\gamma v_{\text {fluid }}
$$


The fluid flow can be produced by effectively flowing the fluid in a microfluidic chamber or, alternatively, by moving the sample stage at a velocity $v_{\text {stage }}$ while keeping the trap fixed, in which case $v_{\text {fluid }}=v_{\text {stage }}$.

A more precise measurement can be obtained by trapping a bead in an optical trap without flow and then oscillating the sample cell holding the liquid that surrounds the trapped bead at a fixed frequency and amplitude. In this case, the resulting equation of motion is the Langevin equation plus a term of the driving force [140]. This PSD consists of the familiar Lorentzian first term, which originates from the Brownian motion of the bead in the harmonic trapping potential, plus a resonance peak at the frequency with which the stage is driven. A similar procedure can be used also with $\mathrm{ACF}$ analysis, where the exponential decay of the thermal fluctuations are superposed to the cosinusoidal oscillations at the stage frequency $[112,147,150]$.

\subsection{Holographic optical tweezers}

The most basic optical tweezers experimental design produces a single optical trap by focusing a single optical beam. Often, however, it is necessary or preferable to handle more than one object simultaneously. For two objects, it is quite easy to split the laser beam to produce two independent traps that can be steered adding movable mechanical parts to the setup. For more than two traps more sophisticated solutions are required. The use of galvanometer-mounted scanning mirrors or acousto-optic-deflectors (AOD) can, partially, solve the problem because these devices are fast enough to move a single beam to the desired positions with a rate much faster than the diffusion of the objects to be trapped, creating so-called Time-Shared Multiple Traps. AODs are much faster than galvomirrors, and by this method it is quite straightforward to create arrays of up to 100 traps. These devices also have the advantage of a high efficiency. The main disadvantage of these devices is that they can trap particles only in the focal plane.

Another common demand is to have non-Gaussian beams such as Bessel or LaguerreGaussian (LG) beams. These have been realised using specialised optical components, e.g. axicons to generate Bessel beams or spiral phase plate for LG beams, or using holographic masks. Nevertheless these components are static and not versatile [151,152].

To overcome these difficulties, it is possible to employ holographic optical trapping (HOT). HOT uses a computer-designed diffractive optical element (DOE) to split a single laser beam into several separate beams, each of which is relayed into an optical tweezers. These optical traps can be made dynamic, and displaced in three dimensions by projecting a sequence of computer- designed holograms. Furthermore, non-Gaussian beam profiles can be encoded in the holographic mask [18].

The working principle of HOT is based on the DOE, whose task is to shape the profile of an incoming optical beam. The DOE is positioned at the front focal plane of the Fourier lens, which collects the first-order diffracted beam, so that the complex amplitude in the back focal plane of the Fourier lens is the Fourier transform of the complex amplitude at the DOE plane $[35,37,153]$.

The easiest way to realise a dynamic DOE is to use a commercially available spatial light modulator (SLM). An SLM is a transmissive or reflective device that is used to spatially modulate the amplitude and/or phase of an optical wavefront in the two dimensions transverse to the beam propagation. SLMs can be programmed to produce light beams with various sculpted optical wavefronts, for instance, they can be used to create optical vortices and higher order Bessel beams, which both have an azimuthal phase variation [154].

HOT are usually realised using reflective devices, nowadays the refresh rates of such devices ranges between about 100 up to $500 \mathrm{~Hz}$ for the fastest device currently available, and 
the reflection efficiency has been greatly enhanced so that it is possible to have a relatively high number of traps with a mid-power DPSS laser.

Once the SLM has been placed in the Fourier plane, to displace the beam in the image plane, the beam at the SLM should be simply deviated with respect to the normal to the SLM plane. To do this, a hologram equivalent to a blazed diffraction grating has to be displayed on the SLM. The angle of the grating determines the direction of the displacement, while the pitch (width of the fringes) determines the displacement angle. Moreover, to displace the focus in the axial direction it is necessary to change the curvature of the wavefront. This is simply accomplished applying to the SLM the phase retardation pattern of a Fresnel lens.

A combination of a diffraction grating and a Fresnel lens therefore produces a shift of the trap in three dimensions. Since the generation of the holographic mask is very fast, this is a very effective way to generate a single optical trap that can be moved in three dimensions in real time.

To generate multiple traps one of the fastest (but also least efficient) algorithms is the random mask encoding algorithm. For every pixel of the SLM, a phase shift is determined as if the hologram was made to generate only one of the $N$ traps. This technique is very fast, and permits one to achieve a good uniformity amongst traps. Nevertheless, the overall efficiency can be very low when the number of traps, $N$, is large, because on average the numbers of pixels that interfere constructively to generate each trap decreases as $1 / N$.

A straightforward algorithm which achieves a better efficiency than the random mask encoding at a just slightly higher computational cost, is the superposition of gratings and lenses algorithm. In this algorithm, the phase of each pixel is chosen to be equal to argument of the complex sum of single-trap holograms. This algorithm has typically a good efficiency at the cost of poor uniformity. In fact, a typical problem that arises using this algorithm is that, when highly symmetrical trap geometries are sought, a part of the energy is diverted to unwanted ghost traps.

To overcome these difficulties it is necessary to use more sophisticated algorithms to compute the phase patterns. The most used are the Gerchberg-Saxton [155] and the adaptiveadditive [156] algorithms, that are able to realise a hologram that will lead to a very close approximation to any desired intensity distribution. The Gerchberg-Saxton algorithm is an iterative algorithm that permits one to find a phase distribution that turns a given input intensity distribution arriving at the hologram plane into a desired intensity distribution in the trapping plane, by propagating the complex amplitude back and forth between these two planes, and replacing at each step the intensity on the trapping plane with the target intensity and that on the SLM plane with the laser's actual intensity profile. The algorithm typically converges after a few tens of iterations, and it is ideally suited to deal with continuous intensity distributions. The adaptive-additive algorithm starts with an arbitrary guess of the phase profile and an initial input wavefront. The Fourier transform of this wavefront is the starting estimate for the output electric field, and the resulting error in the focal plane is reducing by mixing a proportion of the desired amplitude into the field in the focal plane. Inverse Fourier transforming the resulting focal field yields the corresponding field in the input plane. The amplitude in the input plane is replaced with the actual amplitude of the laser profile and finally the algorithm is iterated. The main advantage of this algorithm in comparison to the Gerchberg-Saxton algorithm is that permits one to achieve a better uniformity over all the traps in the array.

The possibility to create multiple dynamic traps and to engineer the wavefront of the trapping laser opened the way to a myriad of new experiment. Holographic optical tweezers have been widely used in many application fields such as optofluidics, optical sorting and the realisation of micromachines $[18,25,157]$. 
Wavefront engineering allowed the realisation of non-Gaussian beams such as HermiteGaussian beams, Laguerre-Gaussian beams, non-diffracting beams and continuous intensity distributions. Such beams find application in optical tweezers for, e.g. controlled rotations of particles using LG beams [158,159], or exploiting the self-healing properties of Bessel beams for multiple trapping [160]. Modifying the laser wavefront allows one to modify also the shape of the energy potential of the trap, this is very important to study transport processes of colloids and macromolecules under the action of thermal forces. Chupeau and colleagues demonstrated that the escape rate can be boosted optimising the potential well shape, even if increasing the barrier height [161].

Holographic optical tweezers have been successfully used to precisely manipulate hybrid block copolymer to create ordered customisable arrays of complex structures speeding up a normally slow processes [162], Shaw and co-workers combined a new photopolymerisation process for rapidly joining simultaneously microspheres assembled in a planar structure by a HOT [163].

Biophysics is one of the fields where HOT have a constant great impact. A new microscopic platform based on holographic optical tweezers has been developed for in vivo applications to study the development of embryos. The setup is able to rapidly measure the viscoelastic properties in different locations of multiple objects held by HOT [164]. Sculpting the shape of the wavefront and dynamically changing it allows to trap, move and control the orientation of non spherical particles. This was used by the group of Halina Rubinsztein-Dunlop to get important information on the properties of swimming cells [165]. The great advantage to held objects far away from surfaces can be combined with advanced imaging technique in addition the ability to rotate and orient trapped cells was used by Diekmann and colleagues to image cross sections of the cells under investigation that are impossible to achieve with conventional sample preparation and immobilisation [166]. The possibility to manipulate a large number of objects simultaneously is of great importance for clinical applications. A research group at Wuhan University modified the surface of polystyrene beads particles in order to selectively adsorb two different liver cancer biomarkers. The particles then were held in a two-dimensional array and investigated using Upconversion Luminescence Encoding technique. The use of HOT greatly improved the sensitivity, specificity and reliability of the biomarkers detection, offering a new alternative for cancer diagnosis technology [167].

Very recently the powerful abilities of HOT have been extended to atomic and molecular physics. In fact optical forces can be exerted also on atoms and molecules to confine and to move them. A titanium sapphire laser tuned far away from atomic resonances was used to trap and to control an array of nine cold rubidium atoms in three dimension in several arrangements [168], Kim and co-workers have implemented a dynamic optical tweezers able to grab, from a cooled cloud, single rubidium atoms and move them in a controlled way for up to $10 \mathrm{~mm}$ [169]. An atom-by-atom assembler has been demonstrated by Barredo et $a l$., they were able to trap simultaneously up to 50 single atoms in a two-dimensional array controlled by an SLM. These works opened exciting prospects for quantum engineering, quantum information and cold chemistry.

\section{Conclusions and outlook}

Optical tweezers are nowadays a key tool for the contactless manipulation of a huge variety of samples. Optical trapping and manipulation is opening new and exciting routes towards the study, characterisation and manipulation of microscopic systems. These techniques have already enabled considerable advances in, e.g. single molecule spectroscopy and novel scan- 
ning probe techniques for surface imaging and force sensing with greatly increased sensitivity. In this review, we have discussed some fundamental aspects of the theory and experimental practice of optical tweezers, providing the readers with a solid starting point from which to explore new potential applications of optical tweezers.

Funding Open access funding provided by Universitá degli Studi di Napoli Federico II within the CRUICARE Agreement.

Open Access This article is licensed under a Creative Commons Attribution 4.0 International License, which permits use, sharing, adaptation, distribution and reproduction in any medium or format, as long as you give appropriate credit to the original author(s) and the source, provide a link to the Creative Commons licence, and indicate if changes were made. The images or other third party material in this article are included in the article's Creative Commons licence, unless indicated otherwise in a credit line to the material. If material is not included in the article's Creative Commons licence and your intended use is not permitted by statutory regulation or exceeds the permitted use, you will need to obtain permission directly from the copyright holder. To view a copy of this licence, visit http://creativecommons.org/licenses/by/4.0/.

\section{References}

1. J. Kepler, De Cometis Libelli Tres (Augustae Vindelicorum, Augsburg, 1619)

2. J.H. Poynting, On the transfer of energy in the electromagnetic field. Phil. Trans. R. Soc. Lond. 175, 343-361 (1884)

3. E.F. Nichols, G.F. Hull, A preliminary communication on the pressure of heat and light radiation. Phys. Rev. 13, 307-320 (1901)

4. P. Lebedev, Untersuchungen über die Druckkräfte des Lichtes. Ann. Phys. 311, 433-458 (1901)

5. R.A. Beth, Mechanical detection and measurement of the angular momentum of light. Phys. Rev. 50, $115-125$ (1936)

6. A. Ashkin, History of optical trapping and manipulation of small-neutral particle, atoms, and molecules. IEEE J. Sel. Top. Quant. Electron. 6, 841-856 (2000)

7. C.H. Townes, How the Laser Happened: Adventures of a Scientist (Oxford University Press, Oxford, 1999)

8. A. Ashkin, Acceleration and trapping of particles by radiation pressure. Phys. Rev. Lett. 24, 156-159 (1970)

9. A. Ashkin, Atomic-beam deflection by resonance-radiation pressure. Phys. Rev. Lett. 25, 1321-1324 (1970)

10. A. Ashkin, J.M. Dziedzic, J.E. Bjorkholm, S. Chu, Observation of a single-beam gradient force optical trap for dielectric particles. Opt. Lett. 11, 288-290 (1986)

11. P.H. Jones, O.M. Maragò, G. Volpe, Optical Tweezers: Principles and Applications (Cambridge University Press, Cambridge, 2015)

12. A. Ashkin, K. Schütze, J.M. Dziedzic, U. Euteneuer, M. Schliwa, Force generation of organelle transport measured in vivo by an infrared laser trap. Nature 348, 346-348 (1990)

13. S.M. Block, L.S.B. Goldstein, B.J. Schnapp, Bead movement by single kinesin molecules studied with optical tweezers. Nature 348, 348-352 (1990)

14. C. Bustamante, J.F. Marko, E.D. Siggia, S. Smith, Entropic elasticity of lambda-phage DNA. Science 265, 1599-1600 (1994)

15. J.T. Finer, R.M. Simmons, J.A. Spudich, Single myosin molecule mechanics: piconewton forces and nanometre steps. Nature 368, 113-119 (1994)

16. C. Bustamante, Z. Bryant, S.B. Smith, Ten years of tension: single-molecule DNA mechanics. Nature 421, 423-427 (2003)

17. K.C. Neuman, A. Nagy, Single-molecule force spectroscopy: optical tweezers, magnetic tweezers and atomic force microscopy. Nat. Methods 5, 491-505 (2008)

18. D.G. Grier, A revolution in optical manipulation. Nature 424, 810-816 (2003)

19. J.E. Molloy, M.J. Padgett, Lights, action: optical tweezers. Contemp. Phys. 43, 241-258 (2002)

20. K. Dholakia, P. Reece, M. Gu, Optical micromanipulation. Chem. Soc. Rev. 37, $42-55$ (2008)

21. A. Jonáš, P. Zemánek, Light at work: the use of optical forces for particle manipulation, sorting, and analysis. Electrophoresis 29, 4813-4851 (2008)

22. M.L. Juan, M. Righini, R. Quidant, Plasmon nano-optical tweezers. Nat. Photon. 5, 349-356 (2011) 
23. K. Dholakia, T. Čižmár, Shaping the future of manipulation. Nat. Photon. 5, 335-342 (2011)

24. M. Padgett, R. Bowman, Tweezers with a twist. Nat. Photon. 5, 343-348 (2011)

25. M. Padgett, R. Di Leonardo, Holographic optical tweezers and their relevance to lab on chip devices. Lab Chip 11, 1196-1205 (2011)

26. P. Polimeno, A. Magazzu, M.A. Iati, F. Patti, R. Saija, C. Degli Esposti Boschi, M.G. Donato, P.G. Gucciardi, P.H. Jones, G. Volpe, O.M. Maragò, Optical tweezers and their applications. J. Quant. Spectrosc. Rad. Transf. 131-150, 2018 (2018)

27. O.M. Maragó, P.H. Jones, P.G. Gucciardi, G. Volpe, A.C. Ferrari, Optical trapping and manipulation of nanostructures. Nat. Nanotech. 8, 807-819 (2013)

28. C. Yi, C.-W. Li, S. Ji, M. Yang, Microfluidics technology for manipulation and analysis of biological cells. Anal. Chim. Acta 560, 1-23 (2006)

29. H. Löwen, Colloidal soft matter under external control. J. Phys. Condens. Matter 13, R415-R432 (2001)

30. D.V. Petrov, Raman spectroscopy of optically trapped particles. J. Opt. A Pure Appl. Opt. 9, S139-S156 (2007)

31. J. Gieseler, J. Millen, Levitated nanoparticles for microscopic thermodynamics—a review. Entropy 20, 326 (2018)

32. L.P. Ghislain, W.W. Webb, Scanning-force microscope based on an optical trap. Opt. Lett. 18, 1678-1680 (1993)

33. E.-L. Florin, A. Pralle, J.H. Hörber, E.H. Stelzer, Photonic force microscope based on optical tweezers and two photon excitation for biological applications. J. Struct. Biol. 119, 202-211 (1997)

34. A.L. Weisenhorn, P.K. Hansma, T.R. Albrecht, C.F. Quate, Forces in atomic force microscopy in air and water. Appl. Phys. Lett. 54, 2651-2653 (1989)

35. E.R. Dufresne, D.G. Grier, Optical tweezer arrays and optical substrates created with diffractive optics. Rev. Sci. Instrum. 69, 1974-1977 (1998)

36. M. Reicherter, T. Haist, E.U. Wagemann, H.J. Tiziani, Optical particle trapping with computer-generated holograms written on a liquid-crystal display. Opt. Lett. 24(9), 608-610 (1999)

37. H. He, M.E.J. Friese, N.R. Heckenberg, H. Rubinsztein-Dunlop, Direct observation of transfer of angular momentum to absorptive particles from a laser beam with a phase singularity. Phys. Rev. Lett. 75, 826829 (1995)

38. N.B. Simpson, L. Allen, M.J. Padgett, Optical tweezers and optical spanners with Laguerre-Gaussian modes. J. Mod. Opt. 43(12), 2485-2491 (1996)

39. B. Piccirillo, S. Slussarenko, L. Marrucci, E. Santamato, The orbital angular momentum of light: genesis and evolution of the concept and of the associated photonic technology. Riv. Nuovo Cimento 36(11), 501-555 (2013)

40. A. Ashkin, Forces of a single-beam gradient laser trap on a dielectric sphere in the ray optics regime. Biophys. J. 61, 569-582 (1992)

41. A. Callegari, M. Mijalkov, A.B. Gököz, G. Volpe, Computational toolbox for optical tweezers in geometrical optics. J. Opt. Soc. Am. B 32(5), B11-B19 (2015)

42. P.C. Chaumet, M. Nieto-Vesperinas, Time-averaged total force on a dipolar sphere in an electromagnetic field. Opt. Lett. 25(15), 1065-1067 (2000)

43. H.C. van de Hulst, Light Scattering by Small Particles (Courier Dover Publications, New York, 1957)

44. G. Gouesbet, G. Gréhan, Generalized Lorenz-Mie Theories (Springer, Berlin, 2011)

45. F. Borghese, P. Denti, R. Saija, Scattering from Model Nonspherical Particles (Springer, Berlin, 2007)

46. E. Higurashi, H. Ukita, H. Tanaka, O. Ohguchi, Optically induced rotation of anisotropic micro-objects fabricated by surface micromachining. Appl. Phys. Lett. 64(17), 2209-2210 (1994)

47. G.A. Swartzlander Jr., T.J. Peterson, A.B. Artusio-Glimpse, A.D. Raisanen, Stable optical lift. Nat. Photon. 5, 48-51 (2010)

48. S.H. Simpson, S. Hanna, T.J. Peterson, G.A. Swartzlander, Optical lift from dielectric semicylinders. Opt. Lett. 37(19), 4038-4040 (2012)

49. M.I. Mishchenko, L.D. Travis, A.A. Lacis, Scattering, Absorption, and Emmision of Light by Small Particles (Cambridge University Press, Cambridge, 2002)

50. B.T. Draine, J. Goodman, Beyond Clausius-Mosotti: wave propagation on a polarizable point lattice and the discrete dipole approximation. Astrophys. J. 405, 685-697 (1993)

51. P.C. Chaumet, M. Nieto-Vesperinas, Electromagnetic force on a metallic particle in the presence of a dielectric surface. Phys. Rev. B 62, 11185-11191 (2000)

52. J.R. Arias-González, M. Nieto-Vesperinas, Optical forces on small particles: attractive and repulsive nature and plasmon-resonance conditions. J. Opt. Soc. Am. A 20(7), 1201-1209 (2003)

53. S. Albaladejo, M.I. Marqués, M. Laroche, J.J. Sáenz, Scattering forces from the curl of the spin angular momentum of a light field. Phys. Rev. Lett. 102, 113602 (2009) 
54. S. Albaladejo, M.I. Marqués, F. Scheffold, J.J. Sáenz, Giant enhanced diffusion of gold nanoparticles in optical vortex fields. Nano Lett. 9, 3527-3531 (2009)

55. D. Gao, W. Ding, M. Nieto-Vesperinas, X. Ding, M. Rahman, T. Zhang, C.-T. Lim, C.-W. Qiu, Optical manipulation from the microscale to the nanoscale: fundamentals, advances and prospects. Light Sci. Appl. 6(9), e17039 (2017)

56. K.Y. Bliokh, A.Y. Bekshaev, F. Nori, Extraordinary momentum and spin in evanescent waves. Nat. Commun. 5, 3300 (2014)

57. C. Triolo, A. Cacciola, S. Patanè, R. Saija, S. Savasta, F. Nori, Spin-momentum locking in the near field of metal nanoparticles. ACS Photon. 4(9), 2242-2249 (2017)

58. M.I. Marqués, Beam configuration proposal to verify that scattering forces come from the orbital part of the poynting vector. Opt. Lett. 39(17), 5122-5125 (2014)

59. G. Volpe, G.P. Singh, D. Petrov, Optical tweezers with cylindrical vector beams produced by optical fibers. Opt. Trapp. Opt. Micromanip. 5514, 283-293 (2004)

60. M.G. Donato, S. Vasi, R. Sayed, P.H. Jones, F. Bonaccorso, A.C. Ferrari, P.G. Gucciardi, O.M. Maragó, Optical trapping of nanotubes with cylindrical vector beams. Opt. Lett. 37, 3381-3383 (2012)

61. S.E. Skelton, M. Sergides, R. Saija, M.A. Iatì, O.M. Maragó, P.H. Jones, Trapping volume control in optical tweezers using cylindrical vector beams. Opt. Lett. 38(1), 28-30 (2013)

62. M.V. Berry, Optical currents. J. Opt. A Pure Appl. Opt. 11(9), 094001 (2009)

63. M. Antognozzi, C.R. Bermingham, R.L. Harniman, S. Simpson, J. Senior, R. Hayward, H. Hoerber, M.R. Dennis, A.Y. Bekshaev, K.Y. Bliokh, F. Nori, Direct measurements of the extraordinary optical momentum and transverse spin-dependent force using a nano-cantilever. Nat. Phys. 12(8), 731 (2016)

64. M.I. Mishchenko, Radiation force caused by scattering, absorption, and emission of light by nonspherical particles. J. Quant. Spectrosc. Radiat. Transf. 70, 811-816 (2001)

65. T.A. Nieminen, H. Rubinsztein-Dunlop, N.R. Heckenberg, A.I. Bishop, Numerical modelling of optical trapping. Comput. Phys. Commun. 142, 468-471 (2001)

66. R. Saija, M.A. Iatí, A. Giusto, P. Denti, F. Borghese, Transverse components of the radiation force on nonspherical particles in the T-matrix formalism. J. Quant. Spectrosc. Rad. Transf. 94, 163-179 (2005)

67. F. Borghese, P. Denti, R. Saija, M.A. Iatì, Optical trapping of nonspherical particles in the T-matrix formalism. Opt. Express 15, 11984-11998 (2007)

68. R.N.C. Pfeifer, T.A. Nieminen, N.R. Heckenberg, H. Rubinsztein-Dunlop, Momentum of an electromagnetic wave in dielectric media. Rev. Mod. Phys. 79, 1197-1216 (2007)

69. F. Borghese, P. Denti, R. Saija, M.A. Iatì, Radiation torque on nonspherical particles in the transition matrix formalism. Opt. Express 14, 9508-9521 (2006)

70. S.H. Simpson, Inhomogeneous and anisotropic particles in optical traps: physical behaviour and applications. J. Quant. Spectrosc. Rad. Transf. 146, 81-99 (2014)

71. T.A. Nieminen, N. du Preez-Wilkinson, A.B. Stilgoe, V.L.Y. Loke, A.A.M. Bui, H. Rubinsztein-Dunlop, Optical tweezers: theory and modelling. J. Quant. Spectrosc. Rad. Transf. 146, 59-80 (2014)

72. S.H. Simpson, S. Hanna, Numerical calculation of inter-particle forces arising in association with holographic assembly. J. Opt. Soc. Am. A 23, 1419-1431 (2006)

73. S.H. Simpson, S. Hanna, Optical trapping of spheroidal particles in gaussian beams. J. Opt. Soc. Am. A 24, 430-443 (2007)

74. S.H. Simpson, D.C. Benito, S. Hanna, Polarization-induced torque in optical traps. Phys. Rev. A 76, 043408 (2007)

75. F. Borghese, P. Denti, R. Saija, M.A. Iatì, O.M. Maragó, Radiation torque and force on optically trapped linear nanostructures. Phys. Rev. Lett. 100, 163903 (2008)

76. R. Saija, P. Denti, F. Borghese, O.M. Maragó, M.A. Iatì, Optical trapping calculations for metal nanoparticles: comparison with experimental data for Au and Ag spheres. Opt. Express 17, 10231-10241 (2009)

77. T.A. Nieminen, V.L. Loke, A.B. Stilgoe, N.R. Heckenberg, H. Rubinsztein-Dunlop, T-matrix method for modelling optical tweezers. J. Mod. Opt. 58, 528-544 (2011)

78. P.C. Waterman, Symmetry, unitarity and geometry in electromagnetic scattering. Phys. Rev. D 3, 825839 (1971)

79. P.L. Marston, J.H. Crichton, Radiation torque on a sphere caused by a circulaly-polarized electromagnetic wave. Phys. Rev. A 30, 2508-2516 (1984)

80. A. Fontes, A.A.R. Neves, W.L. Moreira, A.A. de Thomaz, L.C. Barbosa, C.L. Cesar, A.M. de Paula, Double optical tweezers for ultrasensitive force spectroscopy in microsphere mie scattering. Appl. Phys. Lett. 87, 221109 (2005)

81. A.A.R. Neves, A. Fontes, L.Y. de Pozzo, A.A. de Thomaz, E. Chillce, E. Rodriguez, L.C. Barbosa, C.L. Cesar, Electromagnetic forces for an arbitrary optical trapping of a spherical dielectric. Opt. Express 14, 13101-13106 (2006) 
82. A.A.R. Neves, A. Fontes, L.A. Padilha, E. Rodriguez, C.H. de Brito Cruz, L.C. Barbosa, C.L. Cesar, Exact partial wave expansion of optical beams with respect to an arbitrary origin. Opt. Lett 31, 2477-2479 (2006)

83. G. Gouesbet, G. Gréhan, Generalized Lorenz-Mie Theories (Springer, Berlin, 2011)

84. G. Gouesbet, T-matrix formulation and generalized lorenz-mie theories in spherical coordinates. Opt. Commun. 283, 517-521 (2010)

85. A.A.R. Neves, A. Fontes, C.L. Cesar, A. Camposeo, R. Cingolani, D. Pisignano, Axial optical trapping efficiency through a dielectric interface. Phys. Rev. E 76, 061917 (2007)

86. D.W. Mackowski, Discrete dipole moment method for calculation of the t-matrix for non-spherical particles. J. Opt. Soc. Am. A 19, 881-893 (2002)

87. T.A. Nieminen, H. Rubinsztein-Dunlop, N.R. Heckenberg, Calculation of the T-matrix: general considerations and application of the point-matching method. J. Quant. Spectr. Rad. Transf. 79-80, 1019-1029 (2003)

88. V.L.Y. Loke, T.A. Nieminen, N.R. Heckenberg, H. Rubinsztein-Dunlop, T-matrix calculation via discrete dipole approximation, point matching and exploiting symmetry. J. Quant. Spectrosc. Rad. Transf. 110, 1460-1471 (2009)

89. Y. Cao, A.B. Stilgoe, L. Chen, T.A. Nieminen, H. Rubinsztein-Dunlop, Equilibrium orientations and positions of non-spherical particles in optical traps. Opt. Express 20, 12987-12996 (2012)

90. X. Qi, T.A. Nieminen, A.B. Stilgoe, V.L.Y. Loke, H. Rubinsztein-Dunlop, Comparison of T-matrix calculation methods for scattering by cylinders in optical tweezers. Opt. Lett. 39, 4827-4830 (2014)

91. R. Brown, A brief account of microscopical observations made in the months of June, July and August, 1827 , on the particles contained in the pollen of plants; and on the general existence of active molecules in organic and inorganic bodies. Phil. Mag. 4, 161-173 (1828)

92. E. Nelson, Dynamical Theories of Brownian Motion (Princeton University Press, Princeton, 1967)

93. I. Karatzas, S. Shreve, Brownian Motion and Stochastic Calculus (Springer, New York, 1998)

94. B. Øksendal, Stochastic Differential Equations (Springer, Berlin, 2007)

95. E.M. Purcell, Life at low Reynolds number. Am. J. Phys. 45, 3-11 (1977)

96. W.T. Coffey, Y.P. Kalmykov, The Langevin Equation: With Applications to Stochastic Problems in Physics, Chemistry and Electrical Engineering (World Scientific, Singapore, 2004)

97. G.E. Uhlenbeck, L.S. Ornstein, On the theory of the Brownian motion. Phys. Rev. 36, 823-841 (1930)

98. J. Sethna, Statistical Mechanics: Entropy, Order Parameters, and Complexity (Oxford University Press, Oxford, 2006)

99. S. Jeney, B. Lukić, J.A. Kraus, T. Franosch, L. Forró, Anisotropic memory effects in confined colloidal diffusion. Phys. Rev. Lett. 100(24), 240604 (2008)

100. T. Franosch, S. Jeney, Persistent correlation of constrained colloidal motion. Phys. Rev. E 79(3), 031402 (2009)

101. G. Volpe, L. Helden, T. Brettschneider, J. Wehr, C. Bechinger, Influence of noise on force measurements. Phys. Rev. Lett. 104, 170602 (2010)

102. T. Brettschneider, G. Volpe, L. Helden, J. Wehr, C. Bechinger, Force measurement in the presence of brownian noise: equilibrium-distribution method versus drift method. Phys. Rev. E 83, 041113 (2011)

103. G. Pesce, G. Volpe, G. Volpe, A. Sasso, Long-term influence of fluid inertia on the diffusion of a Brownian particle. Phys. Rev. E 90(4), 042309 (2014)

104. D.L. Ermak, J.A. McCammon, Brownian dynamics with hydrodynamic interactions. J. Chem. Phys. 69(4), 1352-1360 (1978)

105. P. Lançon, G. Batrouni, L. Lobry, N. Ostrowsky, Drift without flux: Brownian walker with a spacedependent diffusion coefficient. EPL (Europhys. Lett.) 54, 28-34 (2001)

106. S. Hottovy, G. Volpe, J. Wehr, Noise-induced drift in stochastic differential equations with arbitrary friction and diffusion in the Smoluchowski-Kramers limit. J. Stat. Phys. 146(4), 762-773 (2012)

107. S. Hottovy, A. McDaniel, G. Volpe, J. Wehr, The Smoluchowski-Kramers limit of stochastic differential equations with arbitrary state-dependent friction. Commun. Math. Phys. 336(3), 1259-1283 (2015)

108. G. Volpe, J. Wehr, Effective drifts in dynamical systems with multiplicative noise: a review of recent progress. Rep. Prog. Phys. 79(5), 053901 (2016)

109. J. Happel, H. Brenner, Low Reynolds Number Hydrodynamics (Spinger, New York, 1983)

110. H. Faxén, Der widerstand gegen die bewegung einer starren kugel in einer zähen flüssigkeit, die zwischen zwei parallelen ebenen wänden eingeschlossen ist. Ann. Phys. 373, 89-119 (1922)

111. G. Volpe, G. Volpe, Simulation of a Brownian particle in an optical trap. Am. J. Phys. 81, 224-230 (2013)

112. G. Pesce, G. Volpe, O.M. Maragò, P.H. Jones, S. Gigan, A. Sasso, G. Volpe, Step-by-step guide to the realization of advanced optical tweezers. J. Opt. Soc. Am. B 32(5), B84-B98 (2015) 
113. S.P. Smith, S.R. Bhalotra, A.L. Brody, B.L. Brown, E.K. Boyda, M. Prentiss, Inexpensive optical tweezers for undergraduate laboratories. Am. J. Phys. 67, 26-35 (1999)

114. J. Bechhoefer, S. Wilson, Faster, cheaper, safer optical tweezers for the undergraduate laboratory. Am. J. Phys. 70, 393-400 (2002)

115. A.S. Mellish, A.C. Wilson, A simple laser cooling and trapping apparatus for undergraduate laboratories. Am. J. Phys. 70, 965-971 (2002)

116. D.C. Appleyard, K.Y. Vandermeulen, H. Lee, M.J. Lang, Optical trapping for undergraduates. Am. J. Phys. 75, 5-14 (2007)

117. W.M. Lee, P.J. Reece, R.F. Marchington, N.K. Metzger, K. Dholakia, Construction and calibration of an optical trap on a fluorescence optical microscope. Nat. Prot. 2, 3225-3238 (2007)

118. M. Mathew, S.I.C.O. Santos, D. Zalvidea, P. Loza-Alvarez, Multimodal optical workstation for simultaneous linear, nonlinear microscopy and nanomanipulation: upgrading a commercial confocal inverted microscope. Rev. Sci. Instrum. 80, 073701 (2009)

119. A.E. Siegman, Lasers (University Science Books, Mill Valley, 1986)

120. P.W. Milonni, J.H. Eberly, Lasers, Wiley Series in Pure and Applied Optics (Wiley, New York, 1988)

121. J.C. Crocker, D.G. Grier, Methods of digital video microscopy for colloidal studies. J. Colloid Interf. Sci. 179, 298-310 (1996)

122. I.F. Sbalzarini, P. Koumoutsakos, Feature point tracking and trajectory analysis for video imaging in cell biology. J. Struct. Biol. 151, 182-195 (2005)

123. S. Helgadottir, A. Argun, G. Volpe, Digital video microscopy enhanced by deep learning, p. 1812.02653 (2008)

124. F. Gittes, C.F. Schmidt, Interference model for back-focal-plane displacement detection in optical tweezers. Opt. Lett. 23(1), 7-9 (1998)

125. B. Lukić, S. Jeney, C. Tischer, A.J. Kulik, L. Forró, E.-L. Florin, Direct observation of nondiffusive motion of a Brownian particle. Phys. Rev. Lett. 95(16), 160601 (2005)

126. T. Franosch, M. Grimm, M. Belushkin, F.M. Mor, G. Foffi, L. Forró, S. Jeney, Resonances arising from hydrodynamic memory in Brownian motion. Nature 478, 85-88 (2011)

127. A. Jannasch, M. Mahamdeh, E. Schaffer, Inertial effects of a small Brownian particle cause a colored power spectral density of thermal noise. Phys. Rev. Lett. 107(22), 228301 (2011)

128. T. Li, S. Kheifets, D. Medellin, M.G. Raizen, Measurement of the instantaneous velocity of a brownian particle. Science 328, 1673-1675 (2010)

129. R.M. Simmons, J.T. Finer, S. Chu, J.A. Spudich, Quantitative measurements of force and displacement using an optical trap. Biophys. J. 70(4), 1813-1822 (1996)

130. I. Chavez, R. Huang, K. Henderson, E.-L. Florin, M.G. Raizen, Development of a fast position-sensitive laser beam detector. Rev. Sci. Instrum. 79(10), 105104 (2008)

131. G. Volpe, G. Kozyreff, D. Petrov, Backscattering position detection for photonic force microscopy. J. Appl. Phys. 102, 084701 (2007)

132. A. Rohrbach, E.H.K. Stelzer, Three-dimensional position detection of optically trapped dielectric particles. J. Appl. Phys. 91(8), 5474-5488 (2002)

133. J.K. Dreyer, K. Berg-Sørensen, L. Oddershede, Improved axial position detection in optical tweezers measurements. Appl. Opt. 43(10), 1991-1995 (2004)

134. J.H.G. Huisstede, V. Subramaniam, M.L. Bennink, Combining optical tweezers and scanning probe microscopy to study dna proteins interactions. Microscop. Res. Tech. 70(1), 26-33 (2006)

135. F.B. Shipley, A.R. Carter, Back-scattered detection yields viable signals in many conditions. Opt. Express 20(9), 9581-9590 (Apr 2012)

136. F. Pierini, K. Zembrycki, P. Nakielski, S. Pawlowska, T.A. Kowalewski, Atomic force microscopy combined with optical tweezers (AFM/OT). Meas. Sci. Technol. 27(2), 025904 (2016)

137. M.J. Lang, C.L. Asbury, J.W. Shaevitz, S.M. Block, An automated two-dimensional optical force clamp for single molecule studies. Biophys. J. 83(1), 491-501 (2002)

138. A. Buosciolo, G. Pesce, A. Sasso, New calibration method for position detector for simultaneous measurements of force constants and local viscosity in optical tweezers. Opt. Commun. 230, 357-368 (2004)

139. K. Berg-Sørensen, H. Flyvbjerg, Power spectrum analysis for optical tweezers. Rev. Sci. Instrum. 75, 594-612 (2004)

140. S.F. Tolić-Nørrelykke, E. Schäffer, J. Howard, F.S. Pavone, F. Jülicher, H. Flyvbjerg, Calibration of optical tweezers with positional detection in the back focal plane. Rev. Sci. Instrum. 77, 103101 (2006)

141. K.C. Vermeulen, J. van Mameren, G.J.M. Stienen, E.J.G. Peterman, C.F. Schmidt, Calibrating bead displacements in optical tweezers using acousto-optic deflectors. Rev. Sci. Instrum. 77(1), 013704 (2006)

142. E.-L. Florin, A. Pralle, E.H.K. Stelzer, J.K.H. Horber, Photonic force microscope calibration by thermal noise analysis. Appl. Phys. A 66, S75-S78 (1998) 
143. N.B. Viana, R.T.S. Freire, O.N. Mesquita, Dynamic light scattering from an optically trapped microsphere. Phys. Rev. E 65, 041921 (2002)

144. G.M. Gibson, J. Leach, S. Keen, A.J. Wright, M.J. Padgett, Measuring the accuracy of particle position and force in optical tweezers using high-speed video microscopy. Opt. Express 16(19), 14561-14570 (2008)

145. G. Volpe, G. Volpe, D. Petrov, Brownian motion in a nonhomogeneous force field and photonic force microscope. Phys. Rev. E 76, 061118 (2007)

146. L.P. García, J.D. Pérez, G. Volpe, A.V. Arzola, G. Volpe, High-performance reconstruction of microscopic force fields from Brownian trajectories. Nat. Commun. 9, 5166 (2018)

147. A. Magazzù, D. Spadaro, M.G. Donato, R. Sayed, E. Messina, C. D’Andrea, A. Foti, B. Fazio, M.A. Iatí, A. Irrera, R. Saija, P.G. Gucciardi, O.M. Maragò, Optical tweezers: a non-destructive tool for soft and biomaterial investigations. Rend. Lincei 26(2), 203-218 (2015)

148. K. Berg-Sorensen, L. Oddershede, E.L. Florin, H. Flyvbjerg, Unintended filtering in a typical photodiode detection system for optical tweezers. J. Appl. Phys. 93(6), 3167-3176 (2003)

149. G. Volpe, D. Petrov, Torque detection using brownian fluctuations. Phys. Rev. Lett. 97, 210603 (2006)

150. M.G. Donato, E. Messina, A. Foti, T.J. Smart, P.H. Jones, M.A. Iati, R. Saija, P.G. Gucciardi, O.M. Marago, Optical trapping and optical force positioning of two-dimensional materials. Nanoscale 10, $1245-1255$ (2018)

151. S.A. Tatarkova, W. Sibbett, K. Dholakia, Brownian particle in an optical potential of the washboard type. Phys. Rev. Lett. 91, 038101 (2003)

152. V. Garbin, D. Cojoc, E. Ferrari, R.Z. Proietti, S. Cabrini, E. Di Fabrizio, Optical micro-manipulation using Laguerre-Gaussian beams. Jpn. J. Appl. Phys. 44(7S), 5773 (2005)

153. J.-M.R. Fournier, M.M. Burns, J.A. Golovchenko. Writing diffractive structures by optical trapping. In S. A. Benton, editor, Practical Holography IX, volume 2406 of Proceedings of the SPIE (1995), pp. $101-111$

154. J.E. Curtis, B.A. Koss, D.G. Grier, Dynamic holographic optical tweezers. Opt. Commun. 207(1), 169175 (2002)

155. R.W. Gerchberg, W.O. Saxton, A practical algorithm for the determination of the phase from image and diffraction plane pictures. Optik 35, 237-246 (1972)

156. E.R. Dufresne, G.C. Spalding, M.T. Dearing, S.A. Sheets, D.G. Grier, Computer-generated holographic optical tweezer arrays. Rev. Sci. Instrum. 72, 1810-1816 (2001)

157. P.H. Jones, O.M. Maragò, G. Volpe, Optical Tweezers: Principles and Applications (Cambridge University Press, Cambridge, 2015)

158. S.H. Simpson, S. Hanna, Rotation of absorbing spheres in Laguerre-Gaussian beams. J. Opt. Soc. Am. A 26(1), 173-183 (2009)

159. S.H. Simpson, S. Hanna, Optical angular momentum transfer by Laguerre-Gaussian beams. J. Opt. Soc. Am. A 26(3), 625-638 (2009)

160. V. Garcés-Chávez, D. McGloin, H. Melville, W. Sibbett, K. Dholakia, Simultaneous micromanipulation in multiple planes using a self-reconstructing light beam. Nature 419, 145-147 (2002)

161. M. Chupeau, J. Gladrow, A. Chepelianskii, U.F. Keyser, E. Trizac, Optimizing Brownian escape rates by potential shaping. Proc. Natl. Acad. Sci. U. S. A. 117(3), 1383-1388 (2020)

162. O.E.C. Gould, S.J. Box, C.E. Boott, A.D. Ward, M.A. Winnik, M.J. Miles, I. Manners, Manipulation and deposition of complex, functional block copolymer nanostructures using optical tweezers. ACS Nano 13(4), 3858-3866 (2019)

163. L.A. Shaw, S. Chizari, R.M. Panas, M. Shusteff, C.M. Spadaccini, J.B. Hopkins, Holographic optical assembly and photopolymerized joining of planar microspheres. Opt. Lett. 41(15), 3571-3574 (2016)

164. F. Hoerner, R. Meissner, S. Polali, J. Pfeiffer, T. Betz, C. Denz, E. Raz, Holographic optical tweezersbased in vivo manipulations in zebrafish embryos. J. Biophoton. 10(11, SI), 1492-1501 (2017)

165. I.C.D. Lenton, D.J. Armstrong, A.B. Stilgoe, T.A. Nieminen, H. Rubinsztein-Dunlop, Orientation of swimming cells with annular beam optical tweezers. Opt. Commun. 459, 124864 (2020)

166. R. Diekmann, D.L. Wolfson, C. Spahn, M. Heilemann, M. Schuettpelz, T. Huser, Nanoscopy of bacterial cells immobilized by holographic optical tweezers. Nat. Commun. 7, 1-7 (2016)

167. C.-Y. Li, D. Cao, C.-B. Qi, Y.-F. Kang, C.-Y. Song, X. Dang-Dang, B. Zheng, D.-W. Pang, H.-W. Tang, Combining holographic optical tweezers with upconversion luminescence encoding: imagingbased stable suspension array for sensitive responding of dual cancer biomarkers. Anal. Chem. 90(4), 2639-2647 (2018)

168. W. Lee, H. Kim, J. Ahn, Three-dimensional rearrangement of single atoms using actively controlled optical microtraps. Opt. Express 24(9), 9816-9825 (2016)

169. H. Kim, W. Lee, H. Lee, H. Jo, Y. Song, J. Ahn, In situ single-atom array synthesis using dynamic holographic optical tweezers. Nat. Commun. 7, 1-8 (2016) 